\title{
Host-associated microbe PCR (hamPCR): accessing new biology through convenient measurement of both microbial load and community composition
}

\author{
Derek S. Lundberg'*, Pratchaya Pramoj Na Ayutthaya', Annett Strauß³, Gautam Shirsekar', \\ Wen-Sui Lo², Thomas Lahaye ${ }^{3}$, Detlef Weigel ${ }^{*}$ \\ 'Department of Molecular Biology, Max Planck Institute for Developmental Biology, 72076 Tübingen, \\ Germany \\ ${ }^{2}$ Department of Evolutionary Biology, Max Planck Institute for Developmental Biology, 72076 Tübingen, \\ Germany \\ ${ }^{3}$ ZMBP-General Genetics, University of Tübingen, Auf der Morgenstelle 32, 72076 Tübingen, Germany \\ *Corresponding authors weigel@tue.mpg.de (D.W.) and derek.lundberg@gmail.com (D.L.)
}

\section{Abstract}

The ratio of microbial population size relative to the amount of host tissue, or "microbial load", is a fundamental metric of colonization and infection, but it cannot be directly deduced from microbial amplicon data such as I6S rRNA gene counts. Because conventional methods to determine load, such as serial dilution plating or quantitative PCR, add substantial experimental burden, they are only rarely paired with amplicon sequencing. Alternatively, whole metagenome sequencing of DNA contributed by host and microbes both reveals microbial community composition and enables determination of microbial load, but host DNA typically greatly outweighs microbial DNA, severely limiting the cost-effectiveness and scalability of this approach. We introduce host-associated microbe PCR (hamPCR), a robust amplicon sequencing strategy to quantify microbial load and describe interkingdom microbial community composition in a single, cost-effective library. We demonstrate its accuracy and flexibility across multiple host and microbe systems, including nematodes and major crops. We further present a technique that can be used, prior to sequencing, to optimize the host representation in a batch of libraries without loss of information. Because of its simplicity, and the fact that it provides an experimental solution to the well-known statistical challenges provided by compositional data, hamPCR will become a transformative approach throughout culture-independent microbiology. 


\section{Introduction}

Knowing the relative abundance of individual taxa reveals important information about any ecological community, including microbial communities. An expedient means of learning their composition in a sample is to sequence and count a defined number of I6S or I8S rRNA genes (hereafter rDNA), the internal transcribed spacer (ITS) of rRNA arrays, or other amplicons that distinguish microbial species in a sample. However, these common amplicon counting-by-sequencing methods do not provide information on the density or load of the microbes. Critically, such microbial sequence counts lack a denominator accounting for the amount of the habitat sampled, and thus, sparsely-colonized and densely-colonized samples become indistinguishable, despite most study systems being open systems in which the total number of microbial cells can vary over many orders of magnitude. Another limitation of such compositional data is that because the sum of all microbes is constrained, an increase in the abundance of one microbe reduces the relative abundance of all other microbes, creating misleading interpretations in the absence of appropriate statistical methods ${ }^{1-4}$. Experimental determination of the microbial load, for example by relating microbial abundance to sample volume, mass, or surface area, has led to important insights in microbiome research that otherwise would have been missed with relative abundance data ${ }^{5-12}$.

For many host-associated microbiome samples, in particular those from plants ${ }^{12}$, nematodes $^{13}$, insects ${ }^{14-16}$, and other organisms in which it is difficult or impossible to physically separate microbes from host tissues, a thorough DNA extraction yields both host and microbial DNA. For such samples, the amount of DNA from host and microbe is directly proportional to the number of cells sampled ${ }^{17,18}$, and therefore the ratio of microbial DNA to host DNA is an intrinsic measure of the microbial load of the sample $e^{6,12,19-21}$. Researchers have attempted to exploit this property and use the host rDNA amplified as a byproduct of microbial rDNA to calculate microbial load ${ }^{6,21}$, but because host nuclear ribosomal arrays may have hundreds or thousands of copies $^{22}$, and organellar DNA is also overabundant, these methods are inefficient and require noisy interventions to increase the microbial signal. Sufficiently deep whole metagenome sequencing (WMS) also can in principle describe the microbial community composition and measure the microbial load, but is rarely practical because of a similar overrepresentation of host DNA ${ }^{12,19}$. For example, WMS of a leaf extract from wild Arabidopsis thaliana typically yields $>95 \%$ plant DNA and $<5 \%$ microbial DNA. Furthermore, many WMS reads remain unclassifiable and thus unquantifiable in complex samples $^{12,19}$.

Most commonly, researchers combine amplicon sequencing with an additional orthogonal method. These include supplementary shallow WMS ${ }^{12}$, quantitative PCR (qPCR) or digital PCR of host and/or microbial genes"16,19,23-26, adding sequenceable "spike-ins" calibrated based on sample volume ${ }^{27}$, mass ${ }^{10}$, or qPCR-determined host DNA content ${ }^{25}$, counting colony 
forming units (CFU) $)^{11,28}$, and flow cytometry ${ }^{5,7}$. The multitude of methods and publications hints at the enduring nature of this problem. While combining amplicon sequencing with any of these other approaches improves data, it requires more work, consumes more sample material, and introduces technical caveats, such as a reliance on accurately pipetting small quantities.

Here, we introduce host-associated microbe PCR or "hamPCR", a robust and accurate single-reaction method to co-amplify a low-copy host gene and one or more microbial regions, such as $16 \mathrm{~S}$ rDNA. We accomplish this with a two-step PCR protoco ${ }^{29-33}$. In hamPCR, gene-specific primer pairs bind to the 'raw' templates in a first short step, which is run for only two cycles to limit propagating amplification biases related to primer annealing and primer availability. In the second exponential step, a single set of primers with complementarity to the universal overhangs add barcodes and sequencing adapters. Such co-amplification of diverse fragments is used in many RNA-seq and WMS protocols ${ }^{34,35}$. Notably, Carlson and colleagues ${ }^{29}$ similarly used a two-step PCR including a multiplexed first step of five to seven cycles to sequence and quantify both variable and joining segments at human $T$ and $B$ cell receptor loci, providing strong proof-of-concept for our method applied to the microbiome.

We designed our host and microbe amplicons to have slightly different lengths, such that they can be resolved by electrophoresis for quality control. We further show that after pooling finished sequencing libraries, the amplicons can be separately purified and re-mixed at any favorable ratio prior for sequencing (for example, with host DNA representing an affordable $5-10 \%$, and sequence counts can be accurately scaled back to original levels in-silico. Thus, in stark contrast to shotgun sequencing, samples with initially unfavorable host-to-microbe ratios can be easily adjusted prior to sequencing without loss of information. Because of the practical simplicity and flexibility of hamPCR, it has the potential to supplant traditional microbial amplicon sequencing in host-associated microbiomes. 


\section{Results}

\section{hamPCR generates quantitative sequencing-based microbial load}

The first 2-cycle "tagging" step of hamPCR multiplexes two or more primer pairs in the same reaction, at least one of which targets a single- or low-copy host gene (Supplementary Figure 1). The tagging primers are then cleaned with Solid Phase Reversible Immobilisation (SPRI) magnetic beads ${ }^{36}$ (Supplementary Figure 2). Next, an exponential PCR of 20-30 cycles is performed using universal barcoded primers (Figure la, Supplementary Figure I). As a host amplicon in $A$. thaliana samples, we targeted a fragment of the GIGANTEA (GI) gene, which is well conserved and present as a single copy in $A$. thaliana and many other plant species ${ }^{37}$. As microbial amplicons, we initially targeted widely-used regions of $16 \mathrm{~S} \mathrm{rDNA}$.

To assess the technical reproducibility of the protocol, we made a titration panel of artificial samples combining varying amounts of pure $A$. thaliana plant DNA with pure bacterial DNA that reflects a simple synthetic community (Methods). These represented a realistic range of bacterial concentrations as previously observed from shotgun metagenomics of wild leaves, ranging from about $0.25 \%$ to $24 \%$ bacterial $D_{N A}{ }^{12}$. We applied hamPCR to the panel, pairing one of three commonly-used 16S rDNA amplicons for the V4, V3V4, and V5V6V7 variable regions with either a 502 bp or 466 bp $G /$ amplicon (Methods, Supplementary Table I), such that the host and microbial amplicons differed by approximately $80 \mathrm{bp}$ in length and were resolvable by gel electrophoresis. In all pairings, the $G /$ band intensity increased as the $16 \mathrm{~S}$ rDNA band intensity decreased (Figure Ib, Supplementary Figure 3).

Focusing on the V4 I6S rDNA primer set, 5I5F - 799R, paired with the 502 bp Gl amplicon, we amplified the entire titration panel in four independently-mixed technical replicates. In addition to use of the chloroplast-avoiding $799 \mathrm{R}$ primer $^{38}$, plant organelle-blocking $\mathrm{PNAs}^{33}$ further prevented unwanted $16 \mathrm{~S}$ rDNA signal from organelles in the pure plant sample (Figure Ib). We pooled the replicates and sequenced them as part of a paired-end HiSeq 3000 lane. Because the $150 \mathrm{bp}$ forward and reverse reads were not long enough to assemble into full amplicons, we analyzed only the forward reads (Methods), processing the sequences into Amplicon Sequence Variants (ASVs) and making a count table of individual ASVs using Usearch ${ }^{39}$. 
bioRxiv preprint doi: https://doi.org/10.1101/2020.05.19.103937; this version posted November 30, 2020. The copyright holder for this preprint (which was not certified by peer review) is the author/funder, who has granted bioRxiv a license to display the preprint in perpetuity. It is made available under aCC-BY 4.0 International license.

Lundberg et al.

Host-associated microbe PCR

a

(1) TAGGING
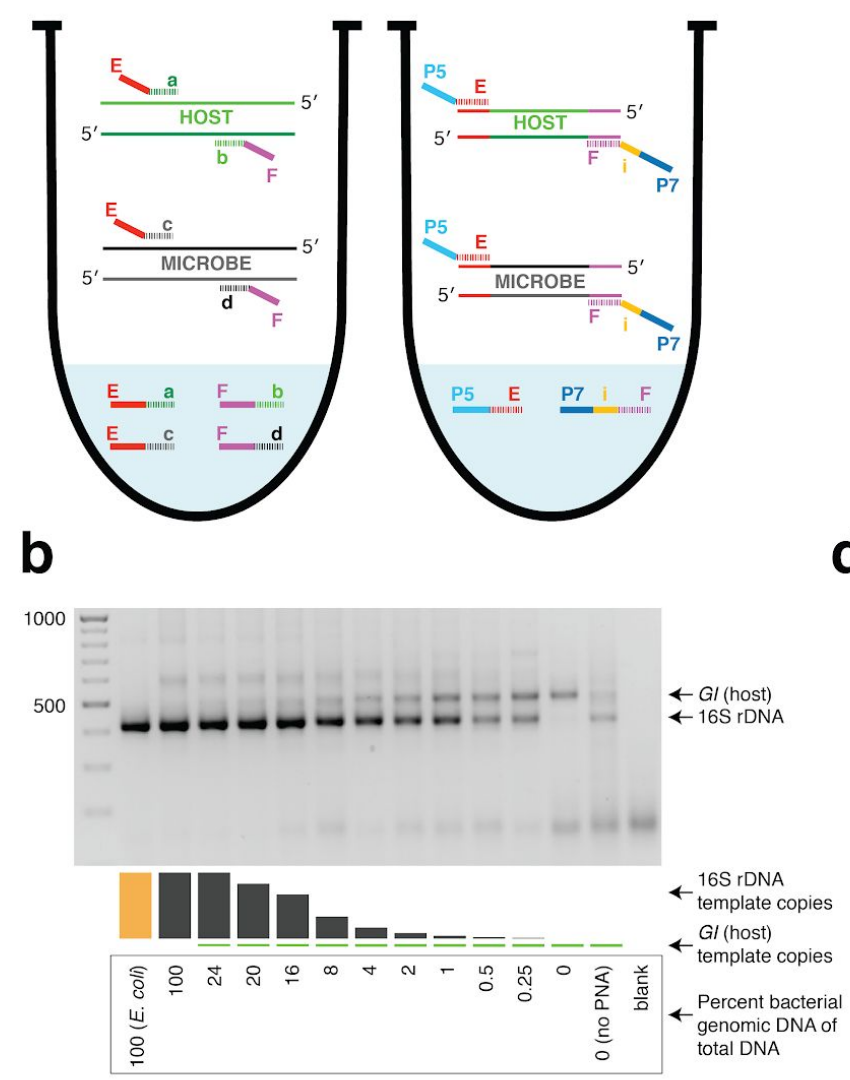

C

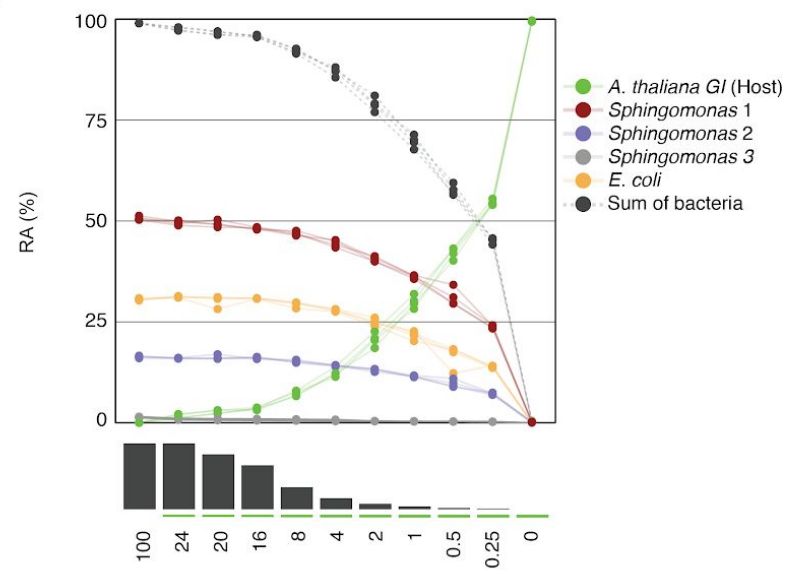

d

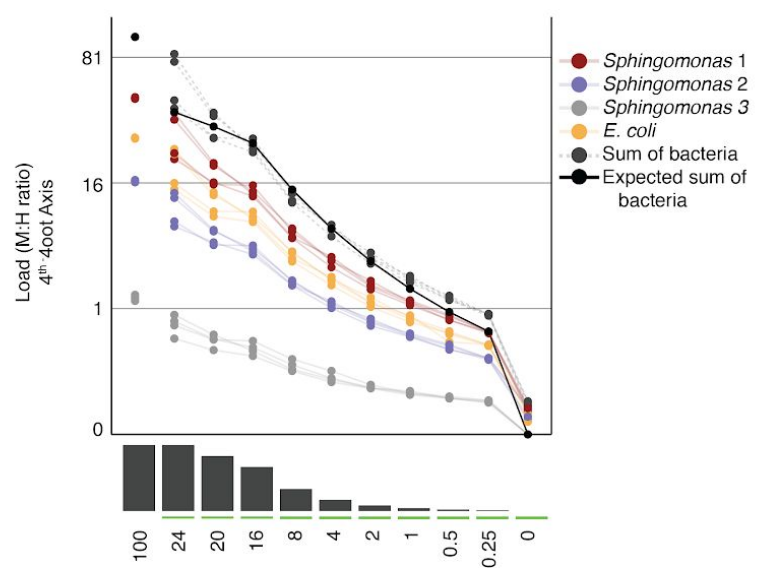

Figure I. Synthetic samples demonstrate technical reproducibility | a, Schematic showing the two steps of hamPCR. The tagging reaction (left) shows two primer pairs: one for the host (E-a and F-b) and one for microbes (E-c and F-d). Each primer pair adds the same universal overhangs $E$ and $F$. The PCR reaction (right) shows a single primer pair (P7-E and P5-i-F) that can amplify all tagged products. $\mathbf{b}$, Representative $2 \%$ agarose gel of hamPCR products from the synthetic titration panel, showing a V4 $16 \mathrm{~S}$ rDNA amplicon at $\sim 420$ bp and an $A$. thaliana $\mathrm{Gl}$ amplicon at $502 \mathrm{bp}$. The barplot underneath shows the predicted number of original G/ and I6S rDNA template copies. Numbers boxed below the barplot indicate the percent bacterial genomic DNA of total DNA. c, Relative abundance of the host and microbial ASVs in the synthetic titration panel, as determined by amplicon counting. Pure E. coli, pure A. thaliana without PNAs, and blanks were excluded. d, Data in (c) converted to microbial load by dividing by host abundance, with a fourth-root transformed $y$-axis to better visualize lower abundances.

After identifying the ASVs corresponding to host $G /$ and the bacteria in the synthetic community, we plotted the relative abundance of $A$. thaliana $G$, the three Sphingomonas ASVs, and the single E. coli ASV across the samples of the titration panel (Figure Ic). There was high consistency between the four replicates, more than what was visually apparent in the gel (Figure Ic, Supplementary Figure 3). We next divided ASV abundances in each sample by the abundance of the host ASV in that sample to give the quantity of microbes per unit of host, a measure of the microbial load. Plotting the data with a fourth-root transformed $Y$ axis, used for better visualization of low bacterial loads, revealed consistent and accurate quantification of 
absolute microbial abundance from 0 up to about 16\% total bacterial DNA (Figure Id). Through this range, the actual sequence counts for total bacteria matched theoretical expectations based on the volumes pipetted to make the titration (solid black line, Figure Id). At $16 \%$ bacterial DNA, bacteria contributed more than $96 \%$ of sequences, and the microbe-to-host template ratio was near 25 . At higher microbial loads the trend was still apparent, and the decrease in precision was likely exacerbated by the effects of small numbers; when the host ASV abundance is used as a denominator and the abundance approaches 0 , load approaches infinity and all sources of error have a greater influence on the quotient. Through simulations, we decided conservatively that loads calculated with a host ASV abundance below $3 \%$ should only be classified as "highly colonized", and should not be used for quantitative measurements (Supplementary Figure 4). In our case, only a minority of highly infected plants ${ }^{12}$ reached bacterial abundances above this highly-quantitative range.

\section{hamPCR does not distort the detected composition of the microbial community}

We amplified the same wild $A$. thaliana phyllosphere template DNA, either with four technical replicates using V4 I6S rDNA primers alone, or alternatively with four technical replicates using hamPCR. After sequencing and deriving ASVs, we first compared ASV abundances within identically-prepared replicates of the pure $16 \mathrm{~S}$ rDNA protocol to demonstrate best-case technical reproducibility of this established technique. As expected, this resulted in a nearly perfect correlation, with a coefficient of determination $\mathrm{R}^{2}$ of 0.99 and abundance distributions as indistinguishable by a Kolmogorov-Smirnov test (Figure 2b). Next, we removed from the hamPCR data the ASV corresponding to $A$. thaliana $G l$, and rescaled the remaining microbial ASVs to $100 \%$ to give relative abundance data. We then compared microbial ASVs from the four pure $16 \mathrm{~S}$ rDNA replicates to those from the four rescaled hamPCR replicates. In this comparison as well, $\mathrm{R}^{2}$ was 0.99 and the distributions were essentially identical (Figure 2c). Thus, the inclusion of a host amplicon in the reaction did not introduce taxonomic biases.

\section{Sensitivity to number of tagging cycles and template concentration}

Two tagging cycles minimize amplification biases that might otherwise have compounding effects due to differential primer efficiencies for the host and microbial templates. However, for templates at low concentrations, inefficiencies due to SPRI cleanup could represent a bottleneck in amplification. Additionally, some techniques that prevent off-target organelle amplification ${ }^{40,41}$ may benefit from additional tagging cycles. To investigate the sensitivity of the results to additional tagging cycles, we applied hamPCR for 2 through 10 tagging cycles, both on the wild $A$. thaliana phyllosphere DNA described above and on a synthetic plasmid-borne template that contains bacterial rDNA and a partial $A$. thaliana $G /$ gene 
bioRxiv preprint doi: https://doi.org/10.1101/2020.05.19.103937; this version posted November 30, 2020. The copyright holder for this preprint (which was not certified by peer review) is the author/funder, who has granted bioRxiv a license to display the preprint in perpetuity. It is made available under aCC-BY 4.0 International license.

template in a $1: 1$ ratio. Surprisingly, for the primers used here, there was no apparent influence of additional tagging cycles, as 7-10 tagging cycles yielded the same distribution of host and 16S rDNA ASV abundances as 2 cycles (Kolmogorov-Smirnov test, $p>0.47$ ). This was true for hamPCR and for $16 \mathrm{~S}$ rDNA primers alone (Figure $2 \mathrm{~d}, 2 \mathrm{e}$ ). This ideal result may not be the case for all primer pairs and should be tested experimentally, but it is consistent with data that either 5 or 7 tagging cycles gave comparable results for quantifying the human immune receptor repertoire ${ }^{29}$, and with the fact that properly-designed multiplex reactions can be used in qPCR carried out with many cycles ${ }^{42}$. We noticed that application of hamPCR to the I:I synthetic template yielded an average of $56.5 \%$ host $G I$ and $43.5 \%$ bacteria, invariant with tagging cycle number (Supplementary Figures 5 and 6). This slight and consistent bias in favor of $G$ may be a result of slight differences in tagging primer efficiency or primer concentration, and should be fine-tunable by altering primer concentration ${ }^{29}$ (Supplementary Figure 7).

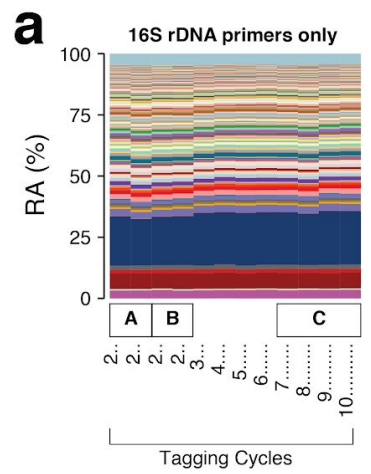

b

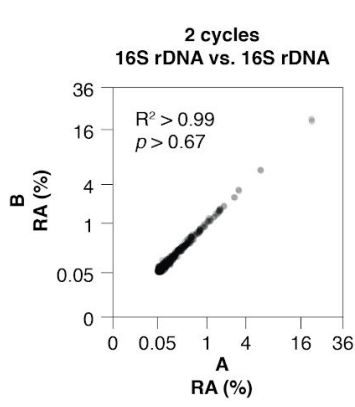

C

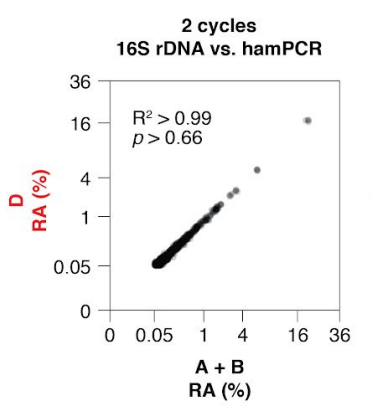

hamPCR
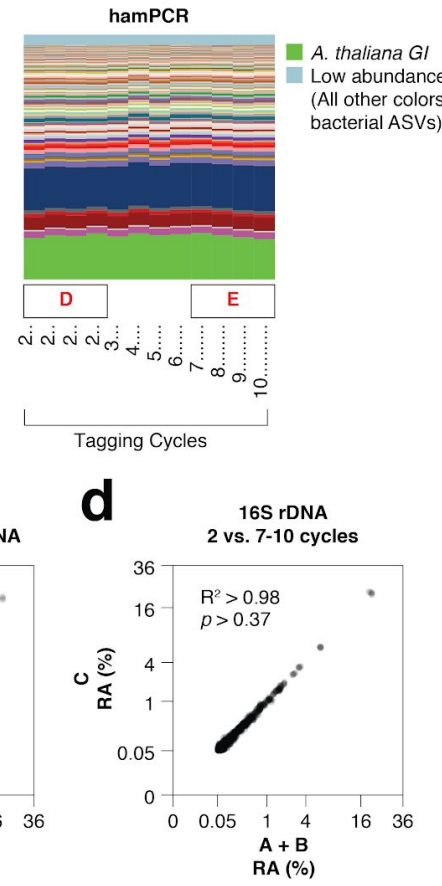

(2) hamPCR

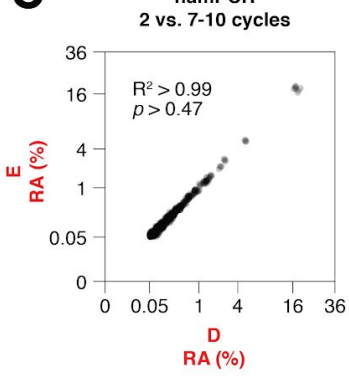

Figure 2. hamPCR is robust and does not distort a complex microbial community | a, DNA extracted from wild $A$. thaliana phyllospheres was used as a template for both V4 I6S rDNA PCR (left, 5I5F and 799R) and hamPCR (right, V4 I6S rDNA and GI 502 bp primers). Four replicates were produced with 2 cycles of the tagging reaction and 30 cycles of PCR, and additional replicates with 3 to 10 tagging cycles paired with 29 to 22 PCR cycles (for a constant total of 32 cycles). The stacked columns show the relative abundances of major ASVs. Boxed upper case letters demarcate groups of samples compared below. b, Correlation of fourth-root transformed ASV abundances for the $16 \mathrm{~S}$ rDNA samples above panel (a) box [A] to the $16 \mathrm{~S}$ rDNA samples above box [B]. Only ASVs with a minimum relative abundance of $0.05 \%$ were compared. $\mathrm{R}^{2}$, coefficient of determination. $p$-value from Kolmogorov-Smirnov test. c, Same as (b), but for the four $16 \mathrm{~S}$ rDNA samples above box $[\mathrm{A}]$ and $[B]$ compared to the four hamPCR samples above box [D]. For hamPCR, the $A$. thaliana GI ASV was removed and the bacterial ASVs were rescaled to $100 \%$ prior to the comparison. d, Same as (b) and (c), but for the four I6S rDNA samples above box $[\mathrm{A}]$ and $[\mathrm{B}]$ compared to the $16 \mathrm{~S}$ rDNA samples above box [C]. e, Same as (b), (c), and (d), but for the four hamPCR samples above box [D] compared to the four hamPCR samples above box $[\mathrm{E}]$. 
bioRxiv preprint doi: https://doi.org/10.1101/2020.05.19.103937; this version posted November 30, 2020. The copyright holder for this preprint (which was not certified by peer review) is the author/funder, who has granted bioRxiv a license to display the preprint in perpetuity. It is made available under aCC-BY 4.0 International license.

Lundberg et al.

Host-associated microbe PCR
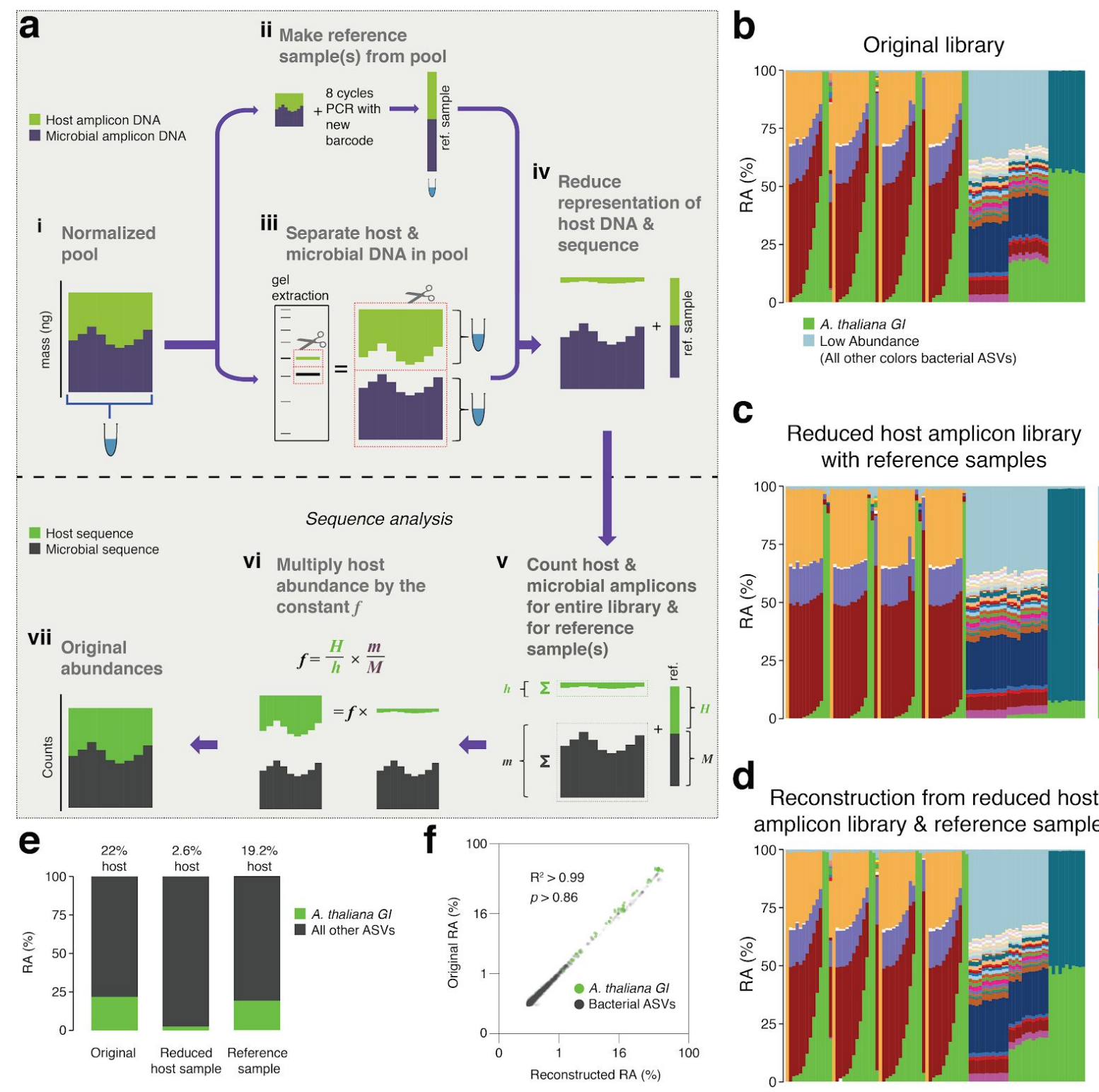

C

Reduced host amplicon library with reference samples

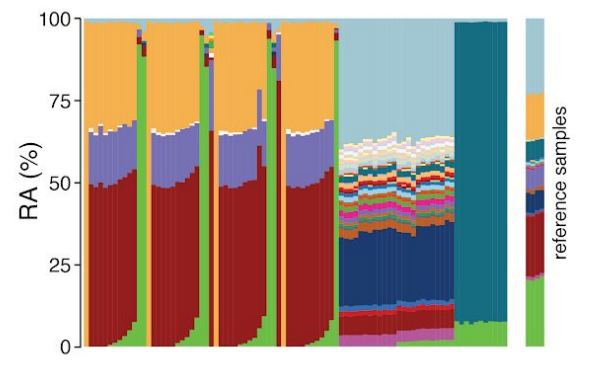

d

Reconstruction from reduced host amplicon library \& reference samples

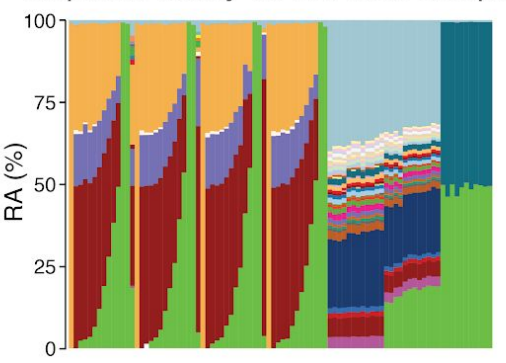

Figure 3. After remixing hamPCR amplicons for efficient sequencing, original abundances can be reconstructed | a, Scheme of remixing process. i: Products of individual PCRs are pooled at equimolar ratios into a single tube. ii: An aliquot of DNA from the pool in (i) is re-amplified with 8 cycles of PCR to replace all barcodes in the pool with a new barcode, creating a reference sample. iii: An aliquot of the pool from (i) is physically separated into host and microbial fractions via agarose gel electrophoresis. iv: The host and microbial fractions and the reference sample are pooled in the ratio desired for sequencing. v: All sequences are quality filtered, demultiplexed, and taxonomically classified using the same parameters. vi: Host and microbial amplicon counts are summed from the samples comprising the pooled library ( $h$ and $m$ respectively), and from the reference sample $(H$ and $M)$. vi: $H, h, M$, and $m$ are used to calculate the scaling constant $f$ for the dataset. All host sequence counts are multiplied by $f$ to reconstruct the original microbe-to-host ratios. vii: Reconstructed original abundances. b, Relative abundance (RA) of actual sequence counts from our original HiSeq 3000 run. c, Relative abundance of actual sequence counts from our adjusted library showing reduced host and 4 reference samples. d, The data from (c) after reconstructing original host abundance using the reference samples. e, The total fraction of host vs. other ASVs in the original library, reduced host library, and reconstruction. $\mathbf{f}$, Relative abundances in the original and reconstructed library for all ASVs with a $0.05 \%$ minimum abundance, shown on $4^{\text {th }}$-root transformed axes. $R^{2}$, coefficient of determination. $p$-value from Kolmogorov-Smirnov test. 
As a further exploration of the robustness of the protocol, we applied hamPCR to a range of total $A$. thaliana leaf template concentrations of between 5 and $500 \mathrm{ng}$ total DNA per reaction, covering a typical template range of 5 to $100 \mathrm{ng}$. Through the typical range, there was no difference in microbe or host ASV abundances. At $200 \mathrm{ng}$ or above, the host amplicon seemed to be slightly favored, possibly because the I6S rDNA primers started to become limiting at these concentrations (Supplementary Figure 8).

\section{Pre-sequencing adjustment of host-to-microbe ratio}

We realized that the size difference between host and microbe bands in hamPCR affords not only independent visualization of both amplicons on a single gel, but also allows convenient and easy adjustment of the host and microbial signals in the pooled library prior to sequencing, in order to improve cost effectiveness. We developed a strategy by which the final hamPCR amplicons are pooled and one aliquot of the pool is rebarcoded to form a reference sample that preserves the original host- to- microbe ratio. The remainder of the pool is run on a gel and the host and microbial bands are separately purified, quantified, and remixed (in our case, to reduce host and gain more microbial resolution). The rebarcoded reference sample, which was not remixed and thereby preserves the original ratio, can be sequenced separately or spiked into the remixed library prior to sequencing. Following sequencing, the reference sample provides the key to the correct host and microbe proportions, allowing simple scaling of the entire library back to original levels (Figure 3a, Supplementary Figure 9).

We made 4 replicate reference samples for our HiSeq 3000 run, which included much of the data from Figure I and Figure 2, and then separately purified the host and microbial fractions of the library (Supplementary Figures 9 and 10). Based on estimated amplicon molarities of the host and microbial fractions, we remixed them targeting $5 \%$ host DNA, added the reference samples, and sequenced the final mix as part of a new HiSeq 3000 lane. A stacked-column plot of relative abundances for all samples on the original run clearly showed the host $A$. thaliana GI ASV highly abundant in some samples, on average responsible for about $22 \%$ of total sequences in the run (Figure $3 \mathrm{~b}, 3 \mathrm{e}$ ). The remixed reduced host library had nearly I0-fold less host GI ASV, 2.6\%, slightly lower than our target of 5\% (Figure 3c, 3e). The reference samples averaged $19.2 \%$ of host GI ASV, very close to the $22 \%$ host fraction in the original library. After using the reference samples to reconstruct the original host abundance in the remixed dataset, we recreated the shape of the stacked column plot from the original library (compare Figure 3d to 3b). When the fourth-root abundances for ASVs above a $0.05 \%$ threshold were compared between the original and reconstructed libraries, the $R^{2}$ coefficient of determination was 0.99 , with no significant difference between the distributions (Kolmogorov-Smirnov test, $p>0.86$ ). 


\section{hamPCR with different I6S rDNA regions compared to shotgun metagenomics}

We next applied hamPCR to leaf DNA from eight wild $A$. thaliana plants that we had previously analyzed by WMS, and from which we therefore had an accurate estimate of the microbial load as the number of microbial reads divided by the number of plant chromosomal reads $^{12}$. We applied hamPCR with primer combinations targeting the host $G /$ gene and either the V3V4, V4, or V5V6V7 variable regions of the I6S rDNA. We produced three independent replicates for each primer set, which we averaged for final analysis (Figure 4, Supplementary Figure II). Across WMS and the three hamPCR amplicon combinations, the relative abundance of bacterial families was consistent (Figure 4a-d: i), with slight deviations likely due to the different taxonomic classification pipeline used for the metagenome reads ${ }^{12}$, as well as known biases resulting from amplification or classification of different I6S rDNA variable regions ${ }^{43}$. After converting both WMS and hamPCR bacterial reads to load by dividing by the plant reads in each sample, we recovered a similar pattern despite the quantification method, with decreasingly lower total loads progressing from plant SI to plant S8, and individual bacterial family loads showing similar patterns (Figure 4a-d: ii, iii). Relative differences in load estimates when comparing the different hamPCR amplicons are likely in part due to different affinities of the I6S rDNA primer pairs for their targets in different bacterial species, and rDNA copy number variation among the microbial families ${ }^{44}$. To quantify the consistency of hamPCR load estimates with WGS load estimates, we plotted the loads against each other and found strong positive correlations, with the highest correlation with the hamPCR using V4 rDNA (Figure $4 \mathrm{e}-\mathrm{g})$.

It is important to note that while relative load ratios between samples were consistent across hamPCR primer sets, the total microbe-to-host ratio varied substantially, with the maximum V5V6V7 I6S rDNA total load at less than 3 times host, and the maximum V4 I6S rDNA total load near 16 times host. This is likely due to variation in GI and I6S rDNA primer efficiencies. To make a statement about the ratio of plant cells to bacterial cells using hamPCR, it would be important to include standard samples with known bacterial load ratios, and to normalize each bacterial taxon by its average rDNA copy number. 
bioRxiv preprint doi: https://doi.org/10.1101/2020.05.19.103937; this version posted November 30, 2020. The copyright holder for this preprint (which was not certified by peer review) is the author/funder, who has granted bioRxiv a license to display the preprint in perpetuity. It is made available under aCC-BY 4.0 International license.

a
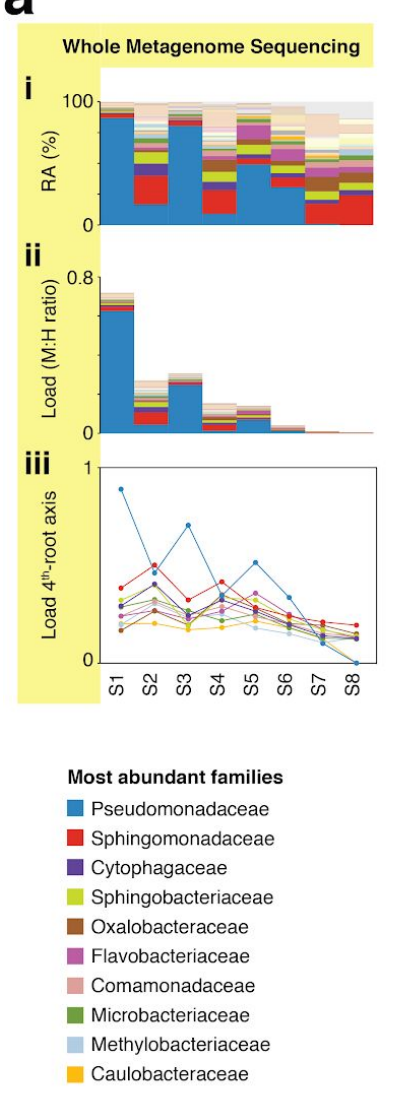

b

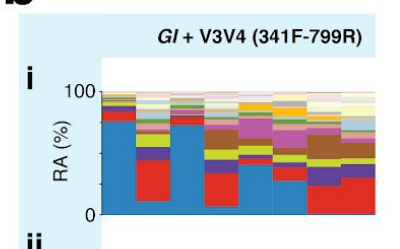

ii
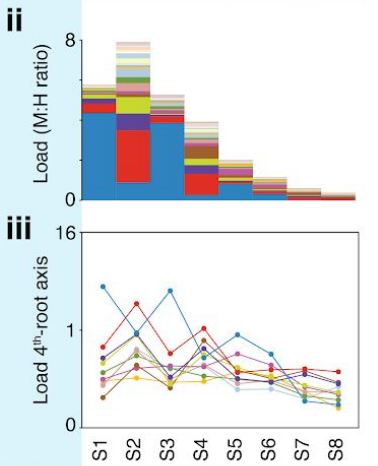

e

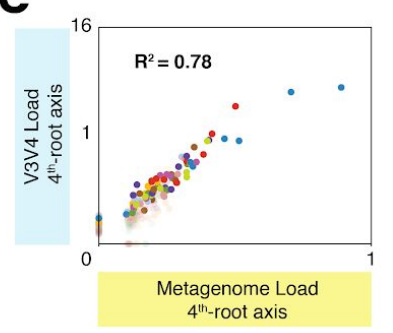

C
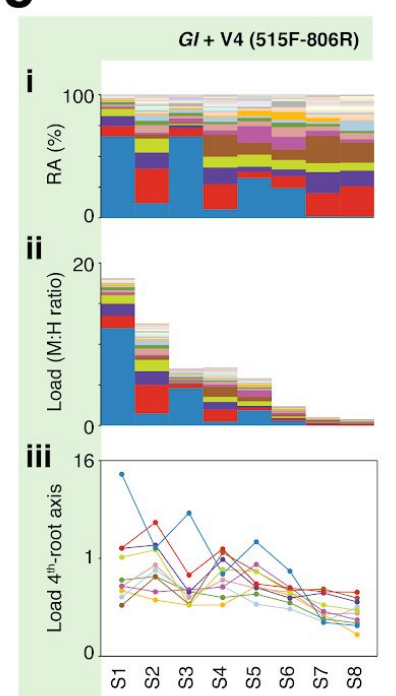

f

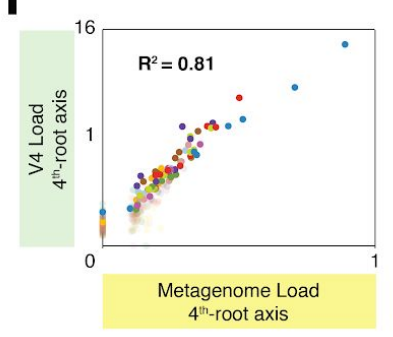

d
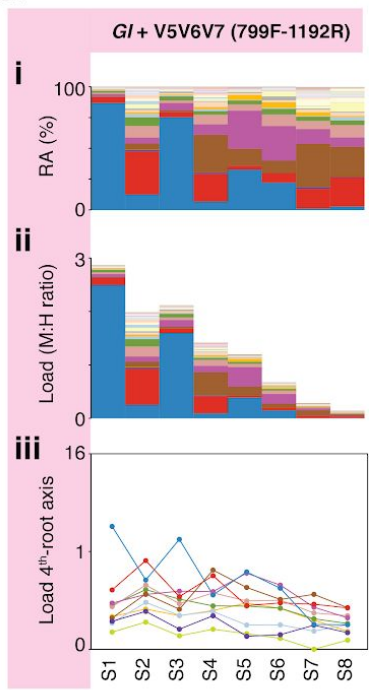

g

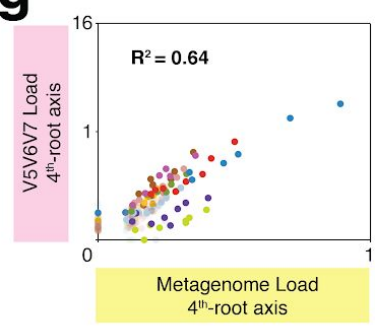

Figure 4. hamPCR with three common I6S rDNA amplicons gives consistent results that agree with shotgun metagenomics | a, i: Stacked-column plot showing the relative abundance (RA) of bacterial families in eight wild $A$. thaliana leaf samples, as determined by shotgun sequencing. The families corresponding to the first 10 colors from bottom to top are shown in reverse order on the bottom left. ii: Stacked-column plot showing the bacterial load of the same bacterial families ( $\mathrm{M}: \mathrm{H}$ ratio = microbe-to-host ratio). iii: The $\mathrm{M}: \mathrm{H}$ bacterial load ratios for the 10 major bacterial families shown on a $4^{\text {th }}$-root transformed $y$-axis. Lines across the independent samples are provided as a help to visualize patterns. b, Similar to (a), but with abundances resulting from hamPCR targeting a 502 bp $A$. thaliana $G$ amplicon and a 290 bp V3V4 I6S rDNA amplicon. c, Similar to (b), but with the I6S rDNA primers targeting a $\sim 420$ bp V4 I6S rDNA amplicon. d, Similar to (b), but with a 466 bp A. thaliana GI amplicon and a $\sim 540$ bp V5V6V7 I6S rDNA amplicon. e, $4^{\text {th }}$ root transformed abundance of each bacterial family determined by hamPCR of V3V4 I6S rDNA plotted against the $4^{\text {th }}$-root transformed bacterial load from shotgun metagenomics. $R^{2}=$ Coefficient of determination. $f$, Same as (e), but for hamPCR of V4 I6S rDNA. g, Same as (e), but for V5V6V7 I6S rDNA.

Three-amplicon hamPCR for simultaneous determination of oomycete and bacterial load

More than two amplicons can be quantitatively tagged and amplified, although it requires more initial troubleshooting to find compatible primers ${ }^{29,30,42,45}$. We set up hamPCR to co-quantify bacteria and eukaryotic oomycetes on plants. These are both diverse microbial groups that include important $A$. thaliana pathogens and that cannot be captured by the same 
rDNA primer set. We first tested universal ITSo primers targeting oomycete rDNA (Methods) in combination with $A$. thaliana $G /$ primers and $16 \mathrm{~S}$ rDNA primers targeting the bacterial $\mathrm{V} 4$, $\mathrm{V} 3 \mathrm{~V} 4$, or V5V6V7 regions, using as template our synthetic plasmid that includes templates for the three primer sets in equal proportion (Methods). A combination of all three amplicons seemed to work efficiently for the V4 region (Supplementary Figure 12), and with this encouraging result, we set up a simple infection experiment. As pathogens, we prepared local strain 466-I of the obligate biotrophic oomycete Hyaloperonospora arabidopsidis $(H p a)^{46}$ and the well-described bacterial pathogen Pseudomonas syringae pv. tomato (Pto) DC3000 ${ }^{47}$. We used two $A$. thaliana genotypes: the reference accession Col-0, which is resistant to Hpa 466-I but susceptible to Pto DC3000, and an enhanced disease susceptibility I (eds I-I) mutant, which has a well-studied defect in a lipase-like protein necessary for many disease resistance responses and which is susceptible to both pathogens ${ }^{48}$.

We infected seedlings with either Hpa 466-I alone, a mix of Hpa 466-I and Pto DC3000, or a buffer control, and maintained them for 7 days under cool, humid conditions ideal for Hpa growth (Methods). The eds I-I plants inoculated with Hpa 466-I became heavily infected and sporangiophores were too numerous to count. No visible bacterial disease symptoms were present on any of the plants, likely because the cool temperature decelerated bacterial growth and symptom appearance ${ }^{49}$. We ground pools of 4-5 seedlings in a buffer and used a small aliquot to count Pto DC3000 CFUs, and the remainder of the lysate for DNA isolation and hamPCR. Despite the lack of bacterial symptoms, we recovered Pto DC3000 CFUs from the inoculated plants.

We applied hamPCR to these samples using the ITSo/I6S/G/ primer set, but due to excessive ITSo product, we repeated library construction replacing the ITSo primers with primers for a single copy Hpa actin gene (Supplementary Figure 12) ${ }^{23}$. Intensity of the actin product correlated with visual Hpa symptoms (Supplementary Figure 12). Sequencing the libraries confirmed $\mathrm{Hpa}$ and Pto ASVs in the inoculated samples, as expected. A standard bacterial relative abundance plot, as would be obtained from pure I6S rDNA data, confirmed the presence of Pto DC3000 in the bacteria-infected samples, and in addition revealed that Hpa-infected samples had a different bacterial community than uninfected samples (Figure 5a). Importantly, it failed to detect obvious differences between microbial communities on Col-0 and eds I-I plants. However, after including the actin ASV from Hpa and converting all abundances to microbial load, a striking difference became apparent between Col-0 and eds I-I, with eds/-I supporting higher bacterial and $\mathrm{Hpa}$ abundances. This is expected from existing knowledge ${ }^{48}$, and supported by Pto DC3000 CFU counts from the same plants (Figure 5b, Figure $5 c$ ). The microbial load plot also revealed that $\mathrm{Hpa-challenged} \mathrm{plants} \mathrm{supported} \mathrm{more}$ bacteria than buffer-treated plants, indicating either that successful bacterial colonizers were unintentionally co-inoculated with $\mathrm{Hpa}$, or that $\mathrm{Hpa}$ caused changes in the native flora (Figure $5 b)$. 
bioRxiv preprint doi: https://doi.org/10.1101/2020.05.19.103937; this version posted November 30, 2020. The copyright holder for this preprint (which was not certified by peer review) is the author/funder, who has granted bioRxiv a license to display the preprint in perpetuity. It is made available under aCC-BY 4.0 International license.

To confirm that the sequence abundances for all three amplicons accurately reflected the concentration of their original templates, we prepared a stepwise titration panel with real samples, mixing increasing amounts of DNA from an uninfected eds/-I plant (low load) into decreasing amounts of DNA from an Hpa-infected eds/-I plant (high load). Sequencing triplicate hamPCR libraries revealed a stepwise increase in ASV levels for all amplicons, consistent with the expectation based on pipetting (Figure $5 \mathrm{~d}$ ). These data, combined with the infection experiment, show that hamPCR is quantitative for at least two independent microbial amplicons in real-world samples.
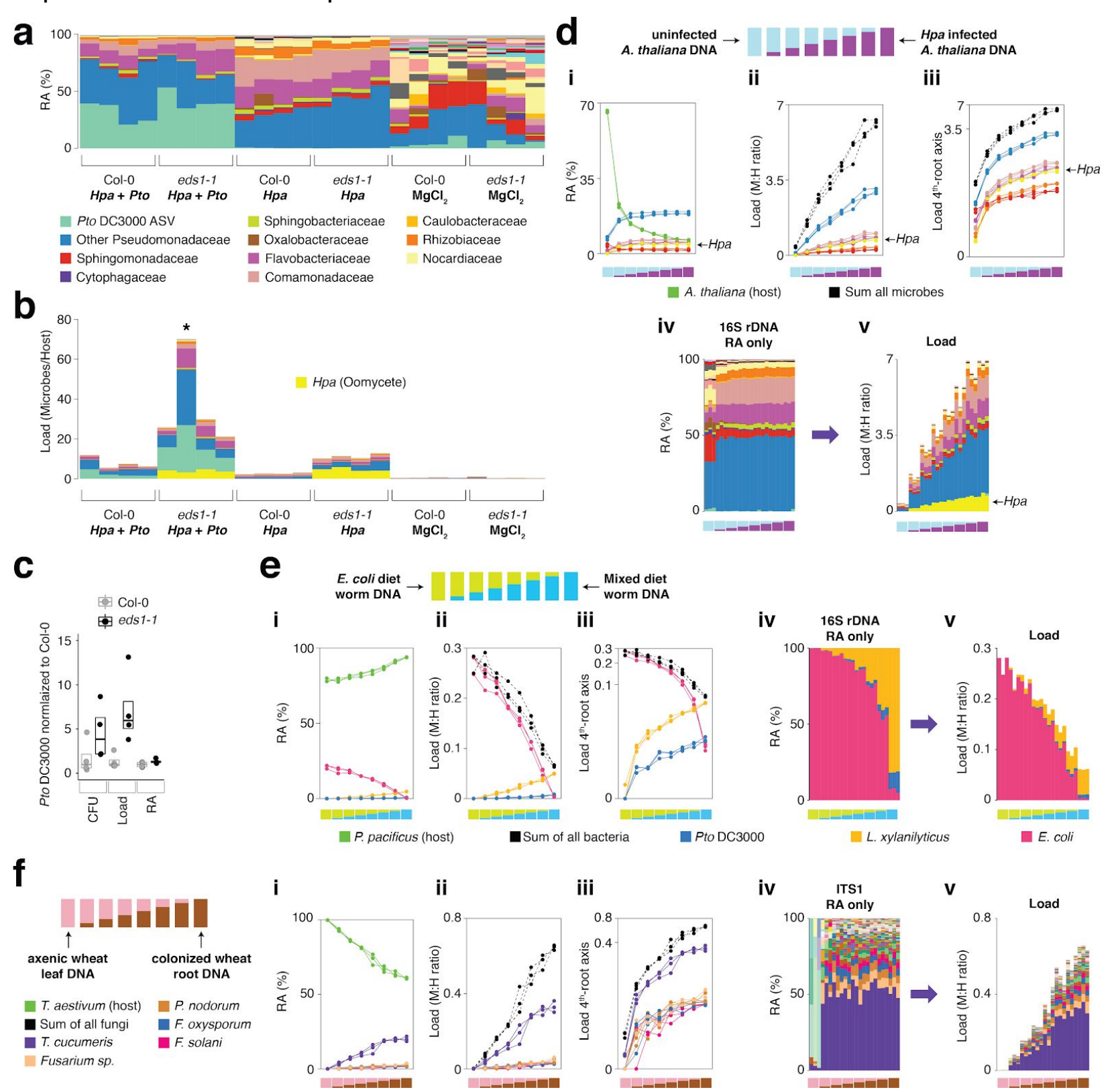

iii
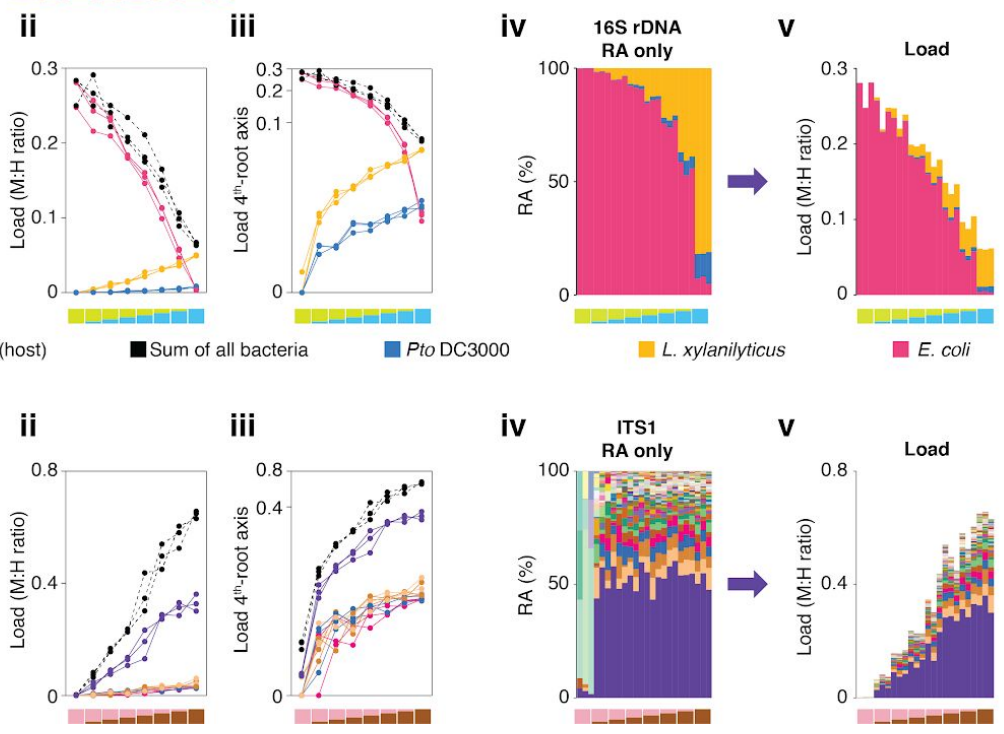

Figure 5. hamPCR can be generalized to more than two amplicons, non-plant hosts, and large host genomes | a, Relative abundance (RA) of only I6S rDNA amplicons for plants co-infected with Hpa and Pto DC3000 and their controls. The ASV corresponding to Pto DC3000 (light green) is shown at the bottom and separately from other Pseudomonadaceae; all other bacteria are classified to the family level, including remaining 
Pseudomonadaceae. b, The same data as shown in (a), but converted to microbial load and with the ASV for Hpa included. The asterisk indicates what is most likely an unreliable load calculation, because host ASV abundance was below $3 \%$ of the total. Same color key as in (a), with an additional color (yellow) for Hpa added. Hpa amplicon abundance was scaled by a factor of 4 in this panel for better visualization. c, Pto DC 3000 bacteria were quantified in parallel on the Col-0 and eds /-I samples infected with Pto DC3000 using CFU counts, the microbial load data in (b), or the relative abundance data in (a). The median is shown as a horizontal line and box boundaries show the lower and upper quartiles. d, An uninfected plant sample was titrated into an $H$ pa-infected sample to make a panel of eight samples. i: the relative abundance of hamPCR amplicons with median abundance above $0.15 \%$. ii: after using host ASV to convert amplicons to load. The cumulative load is shown in black. iii: the load on a $4^{\text {th }}$-root transformed $y$-axis, showing less-abundant families. iv: stacked column visualization of all ASVs for the panel as it would be seen with pure $16 \mathrm{~S}$ rDNA data. v: stacked-column plot of the panel corrected for microbial load. Same color key as in (b), but with colors for A. thaliana and sum of microbes added. e, Similar to (d), but with the nematode worm $P$. pacificus as host, and V5V6V7 I6S rDNA primers. Instead of bacterial families, specific ASV abundances are shown. f, Similar to (e), but with hexaploid wheat $T$. aestivum as host, and fungal ASV abundances from ITSI amplicons.

\section{Utility in diverse hosts and crops with large genomes}

To demonstrate the utility of hamPCR outside of plants, we prepared samples of the nematode worm Pristionchus pacificus, fed on a diet of either pure E. coli OP50, or alternatively a mix of E. coli OP50 with Pto DC3000 and Lysinibacillus xylanilyticus. The worms were washed extensively with PBS buffer to remove epidermally-attached bacteria, enriching the worms for gut-associated bacteria, and we prepared DNA from each sample. In the same manner as described in the previous section, we titrated the two DNA samples into each other to create a panel of samples representing a continuous range of colonization at biologically-relevant levels. Over three replicates, hamPCR accurately captured the changing bacterial loads of the gut microbes (Figure 5e, Supplementary Figure 13). We similarly validated the technique for fungal and bacterial microbes of Triticum aestivum (bread wheat) the most widely grown crop in the world and one of the most difficult to study due to a 16 Gb haploid genome size ${ }^{50}$. To simulate different levels of infection, we titrated DNA from axenically-grown wheat leaves into DNA from wheat roots that had been cultivated in non-sterile soil and applied hamPCR, using as a host gene RNA polymerase $\mathrm{Al}$ (PolAl), which is present as a single copy in each of the $A, B$, and $D$ subgenomes ${ }^{51}$. We recovered expected abundance patterns in the panel both for ITSI rDNA primers (Figure 5f, Supplementary Figures I4 and I5) and for V4 I6S rDNA primers (Supplementary Figures 14 and 15). We noticed the original ratio of ITSI to PolAI sequences recovered was low; because ITS primers produce amplicons that are highly variable in length, some of which may co-migrate with the host amplicon on a gel, the cut-and-mix approach described in Figure 3 could not be used to improve ITSI representation. However, increasing the ratio of the ITSI:PoIAI tagging primers from I:I to 2:I (Methods) successfully enriched the ITSI amplicon without sacrificing relative load determination between samples (Supplementary Figures 14 and 15). 
To demonstrate the ability of hamPCR to yield new biological insights into complex study systems, we conducted two experiments with crop plants. First, we set up a growth curve in bell pepper (Capsicum annuum), which has a $3.5 \mathrm{~Gb}$ genome ${ }^{52}$, approximately $25 \times$ larger than $A$. thaliana, and the pepper pathogenic bacterium Xanthomonas euvesicatoria $(X e)$ strain $85-10^{53}$. As proof-of-concept preparation for the growth curve, to confirm that hamPCR could accurately capture absolute changes in pathogen abundance in pepper leaves, we constructed an infiltration panel in which $X e$ 85- 10 was diluted to final concentrations of $10^{4}$, $10^{5}, 10^{6}, 10^{7}$ and $10^{8} \mathrm{CFU} / \mathrm{mL}$ and infiltrated into four replicate leaves per concentration. Immediately afterwards, without further bacterial growth, we harvested leaf discs within inoculated areas using a cork borer. We ground the discs and used some of the lysate for $X e$ 85- 10 CFU counting, and the remainder for DNA extraction and hamPCR targeting the V4 I6S rDNA and the pepper $\mathrm{Gl}$ gene ( $\mathrm{CaGl}$ ), and qPCR, targeting the xopQ gene for a Xe type III effector ${ }^{54}$, and the $C$. annuum $U B /-3$ gene for a ubiquitin-conjugating protein ${ }^{55}$.

Sequencing the hamPCR libraries revealed that as the $X e$ 85-10 infiltration concentration increased, so did the resulting load of the ASV corresponding to $\mathrm{Xe}$ 85-10 (Supplementary Figure 16 and 17a). The other major bacterial classes detected in the infiltration panel, comprising commensal bacteria already present in the leaves, had similar, low abundances, regardless of the amount of infiltrated Xe 85-10 (Supplementary Figure 17b). When aligned with CFU counts recovered from the same lysates (Methods), the hamPCR Xe 85-10 ASV loads showed nearly the same exponential differences between samples, although at lower infiltration concentrations, $\mathrm{QPCR}$ and hamPCR gave a slightly higher estimate than CFU counts (Supplementary Figure 17c). The presence of a low level of native, antibiotic-sensitive $X e$ on the leaves could potentially explain this discrepancy, because this could be detected by DNA-based methods but not culturing.

For the pepper growth-curve, we infiltrated six $C$. annuum leaves of six different plants with $X e$ 85-10 at a concentration of $10^{4} \mathrm{CFU} / \mathrm{mL}$, and took samples from each plant at 0, 2, 4, 7, 9 and II dpi for CFU counting, qPCR, and hamPCR. We observed a rapid increase in $X e$ 85-I0 ASV abundance as a result of rapid bacterial growth, leveling off at $7 \mathrm{dpi}$ (Figure 6a). By 7 dpi, bacterial growth had reduced the host $\mathrm{Gl}$ amplicon abundance to below $3 \%$, making the load corrections unreliable from day 7 on (gray box, Figure 6a). Aligned Xe 85-10 ASV loads compared very closely to CFU counts and to aligned qPCR abundances up to $7 \mathrm{dpi}$ (Figure 6c). Notably, the other major bacterial classes, Actinobacteria and the Alpha-, Beta-, and Gammaproteobacteria, also increased in microbial load through time, a trend significant even comparing $2 \mathrm{dpi}$ to $0 \mathrm{dpi}$ (Figure 6b, Mann-Whitney U-test, $p<0.00 \mathrm{I}$ ). This increase in load for the other classes was not a PCR artifact due to high $X e$ 85-10 titers, because in the infiltration panel, measurements for these classes had not changed even at higher pathogen concentrations (Supplementary Figure 17b). This subtle but biologically significant effect of infection on growth of commensal bacteria would be completely invisible in a pure $16 \mathrm{~S}$ rDNA amplicon analysis, which would only show $X e 85-10$ overtaking the community. 
bioRxiv preprint doi: https://doi.org/10.1101/2020.05.19.103937; this version posted November 30, 2020. The copyright holder for this preprint (which was not certified by peer review) is the author/funder, who has granted bioRxiv a license to display the preprint in perpetuity. It is made available under aCC-BY 4.0 International license.

Lundberg et al.

Host-associated microbe PCR

a

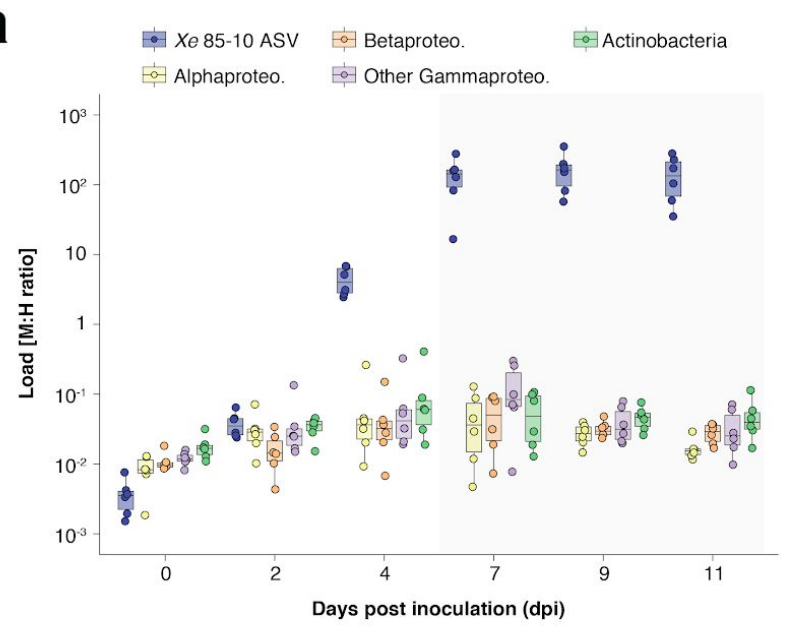

b

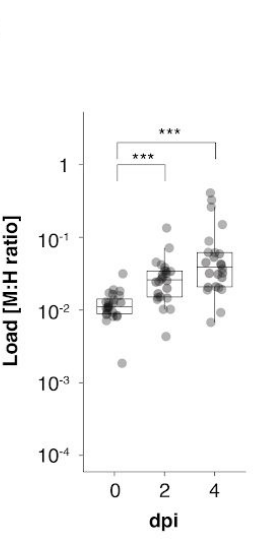

C

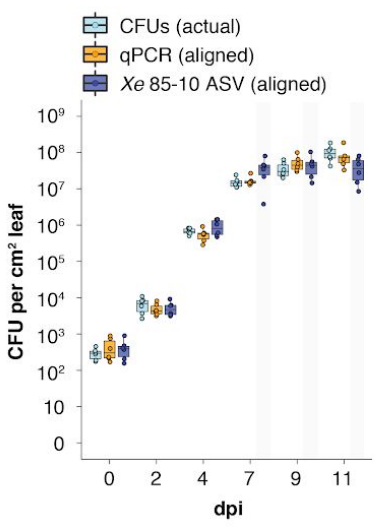

d

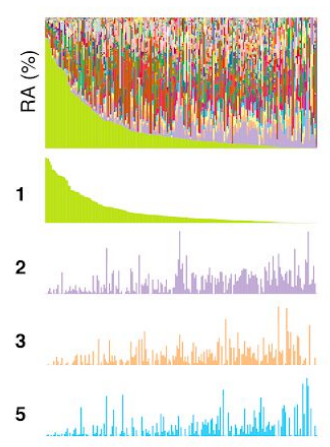

e
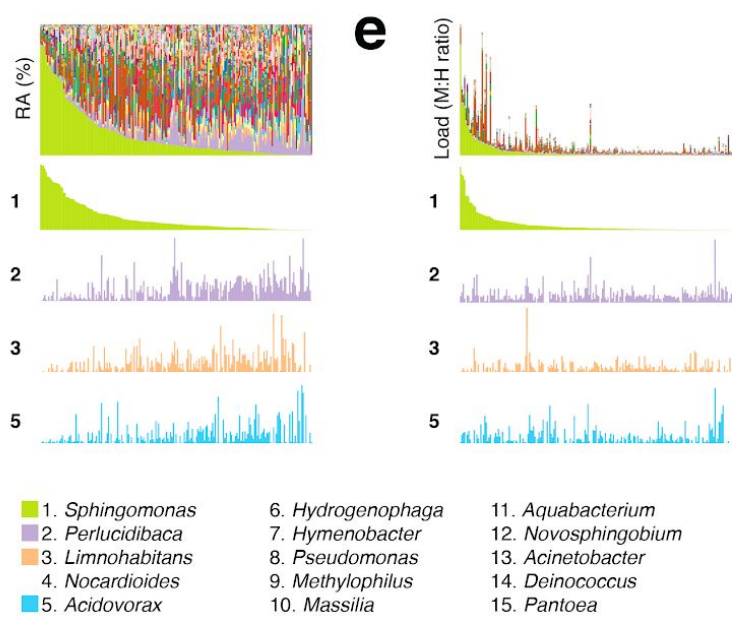

f
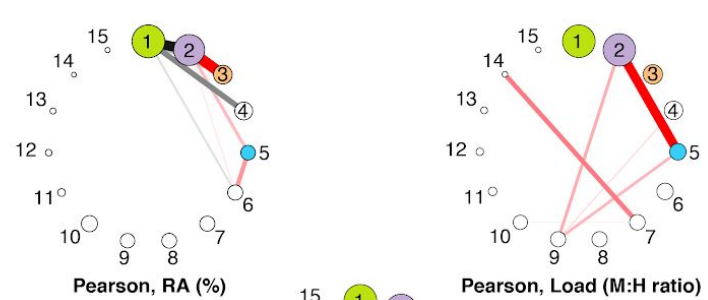

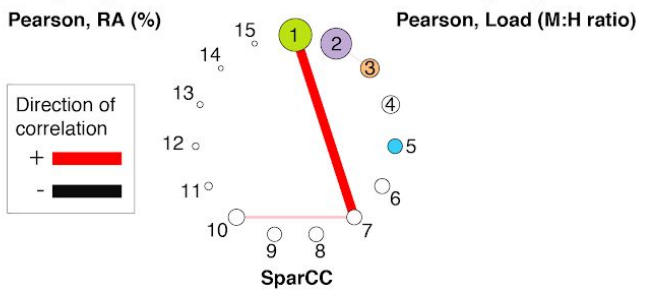

Figure 6. hamPCR can provide new insights into microbial interactions in crop plants | a-c, $C$. annuum growth curve experiment. All y-axes are on a base- 10 logarithmic scale. In all boxplots, the median is represented by a horizontal line and box boundaries show the lower and upper quartiles. Whiskers extend from the box up to I.5 times the interquartile range. a, $X e$ 85- 10 was inoculated into $C$. annuum leaves at $10^{4} \mathrm{CFU} / \mathrm{mL}$. Leaf samples were taken at $0,2,4,7,9$, and II days post inoculation (dpi), and hamPCR performed. The corrected load is shown for the particular ASV corresponding to $X e$ 85-10, as well as for the major bacterial classes. $\mathbf{b}$, The total load for all bacterial classes shown in (d) at 0,2 , and $4 \mathrm{dpi}(* * *=p<0.00 \mathrm{I})$. c, Actual CFU counts for Xe 85-10 in the growth curve experiment juxtaposed with aligned qPCR and hamPCR loads. d-e, Field-grown $Z$. mays collection. d, Relative abundance (RA) of bacterial genera found in $Z$. mays leaf hole punches, ordered by Sphingomonas relative abundance. The genera corresponding to the first 15 colors from bottom to top are shown in reverse order in the legend. The relative abundance of four isolated genera is highlighted (colored boxes in legend). e, same as $\mathbf{d}$, but showing microbial load rather than relative abundance and ordered by Sphingomonas load. $\mathbf{f}$, Correlation networks of the same 15 genera from the legend for $\mathbf{d}$ and $\mathbf{e}$. Pearson correlation from RA data from d (left), pearson correlation of microbial load from e (right), and SparCC correlation network (bottom). Circles representing genera are scaled such that their area represents the median genus abundance across all samples. Only correlations of absolute magnitude $>=0.3$ are shown.

Finally, we applied hamPCR to DNA from 20I leaf samples from mature, isogenic maize (B73) growing in a field site in Tübingen, Germany. We used the V4 region of $16 \mathrm{~S}$ rDNA for bacteria and the single copy LUMINIDEPENDENS (LD) gene as a host marker, and plotted both the relative abundance of bacterial genera (Figure 6d) and the bacterial load of these genera (Figure 6e). In some samples, the genus Sphingomonas exceeded $80 \%$ of the bacterial 
community, creating especially strong compositionality effects; other abundant genera Perlucidibaca, Limnohabitans, and Acidovorax visibly increased in relative abundance as Sphingomonas became less abundant (Figure 6d). In contrast, the bacteria load of these same genera appeared mostly unaffected by Sphingomonas bacterial load (Figure 6e). As expected, a Pearson correlation network made with relative abundance data revealed that Sphingomonas was negatively correlated with many genera, a well-known and problematic artifact of compositionality $^{56}$ (Figure 6f). A Pearson correlation network made with microbial load data was remarkable in that Sphingomonas, despite having the highest median abundance of any genus, is among the genera least correlated with others (Figure 6f). We also calculated a correlation network using SparCC ${ }^{56}$, which estimates Pearson correlations on log-transformed components to avoid compositionality artifacts. This network did indeed avoid the spurious negative correlations with Sphingomonas, although it still implicated the genus more strongly than the true correlation network built with hamPCR data. Each network has a very different biological interpretation. If Sphingomonas abundance does not influence these other genera on healthy leaves, this could mean, for example, that it can colonize more of the available extreme habitat, with its success determined largely by abiotic factors. Future study will be necessary to resolve this. Overrepresentation of Sphingomonas is a feature shared by other major studies of the maize leaf microbiome ${ }^{57,58}$; overcoming this compositionality problem is broadly relevant to studies of this microbial habitat.

\section{Discussion}

We developed hamPCR, a simple and robust method to quantitatively co-amplify one or more microbial marker genes along with an unrelated host gene, allowing accurate determination of microbial load and microbial community composition from a single sequencing library (Figure I, Figure 2). Furthermore, we developed a method to predictably optimize the amount of sequencing effort devoted to microbe vs. host, without losing information about the original microbe to host ratio (Figure 3). This is an important advance in our approach that greatly increases cost-efficiency.

The principle behind hamPCR stands on a body of literature describing related, firmly established techniques, which bodes well for wide-spread adoption of our approach. Using two steps in a PCR protocol is common in amplicon sequencing, including of microbial marker genes $^{31-33,59,60}$. Two-step PCR protocols provide the major advantage that only a one-time investment is needed in a set of universal barcoding primers for a flexible step two. These can be easily adapted to any amplicon(s) by simply swapping in different template-specific primers for step one. For labs already equipped for two-step PCR, implementing hamPCR involves only slight adjustments to cycling conditions and template-specific tagging primers.

Quantitative co-amplification using multiple primer pairs also has proven reliable ${ }^{29,30,61}$, and PCR biases affecting co-amplification of diverse fragments are manageable and 
well-understood from popular RNA-seq and WMS protocols ${ }^{62,63}$. This rich literature should increase confidence when implementing hamPCR in microbiome research, and it also provides resources for optimization and further development. For example, the use of fewer cycles in exponential PCR could reduce noise and bias, hamPCR tagging primers could be fitted with UMls for higher precision, and the protocol could be adapted for sequencing platforms with longer read lengths.

We have demonstrated that microbial load measurement is sensitive to the relative concentrations between the host and microbe primers in the tagging step (Supplementary Figure 7), consistent with the effects of primer concentration on amplification efficiency in qPCR ${ }^{64-66}$. This property makes it possible to fine-tune the primer ratios, either to yield the expected ratio of products ${ }^{29}$, or to intentionally increase the representation of a microbial amplicon for more efficient sequencing (Supplementary Figure 16a). The effect of primer concentration has important implications for how a large project should be prepared. We recommend that the tagging primers be carefully pipetted into a multiplexed primer master mix sufficiently large to be used for the entire project, or alternatively the same control samples should be sequenced across sample batches to allow correction of slight batch differences.

A limitation of hamPCR is reduced accuracy at the highest microbial loads (Supplementary Figure 4). Only a minority of our samples reached a level of infection that interfered with accurate quantification, and we expect that this will be the case for most colonized hosts. If not, there are three straightforward adjustments that can increase host signal to acceptable levels. First, altering the host and microbe amplicon ratio in the pooled library prior to sequencing, as demonstrated in Figure 3, could be used to increase the overall host representation. Second, a host gene with a higher copy number could be chosen for template tagging throughout the entire project. Finally, adjusting the concentration of the host primers in the tagging reaction could also increase the representation of host (Supplementary Figure 7) ${ }^{29}$.

In summary, we have demonstrated that hamPCR is agnostic to the taxonomic identities of the organisms studied on both the host and microbe side, their genome sizes, or the functions of the regions amplified. We have also shown that hamPCR can monitor three amplicons at the same time for interkingdom microbial quantification, and in principle can multiplex more with careful design. Our focus here has been on tracking hosts and their closely-associated microbes, but the protocol could also be adapted to quantitatively relate different amplicons targeting archaea, bacteria, and fungi in diverse "host-free" environments like soil. Besides whole organisms, hamPCR also enables quantitative monitoring of bacterial populations and sub-genomic elements, such as plasmids or pathogenicity islands that might not be shared by all strains in a population. An exciting application of hamPCR is the study of endophytic microbial colonization and infection in crop plants, many of which have very large genomes that preclude the analysis of any sizable number of samples by shotgun sequencing. In a previous study, we sequenced leaf metagenomes from over 200 A thaliana plants, at not insignificant costs ${ }^{12}$. In wheat, assuming comparable microbial loads, the same investment in 
sequencing would barely be sufficient for two samples due to the size of the wheat genome of over $16 \mathrm{~Gb}$. Microbial analysis of the $>200$ samples we processed of field-grown maize likewise would be prohibitively expensive by shotgun sequencing, and supplementing these data with an orthogonal method on this scale requires at least double the sample and double the time.

Other exciting applications are the recognition of cryptic infections ${ }^{67}$, tracking of mixed infections, and measurement of pathogen abundances on hosts showing quantitative disease resistance - this could even be accomplished by spiking hamPCR amplicons into the same sequencing run used to genotype the hosts ${ }^{68}$. In sparsely-colonized samples, hamPCR will help prevent inflating the abundance of ultra-low abundance microbes, such as reagent contaminants. Finally, for projects with many samples, the fact that hamPCR derives microbial composition and load from the same library not only saves costs and uses less of the sample, but also simplifies analysis and project organization. 


\section{Methods}

\section{hamPCR protocol}

hamPCR requires two steps: a short 'tagging' reaction of 2 cycles, and a longer 'exponential' reaction. We used 30 cycles throughout this work, although fewer can and should be used if the signal is clear for better quantitative results. The primers employed in the tagging reaction were used at $1 / 8$ the concentration of the exponential primers, as this still represents an excess in a reaction run for only two cycles, prevents waste, and reduces dimer formation. See Supplementary Information and Supplementary Table I for detailed information about the primers.

\section{Tagging reaction}

We used Taq DNA Polymerase (NEB, Ipswich, MA, USA) for the first tagging step, and set up $25 \mu \mathrm{L}$ reactions as follows.

Master Mix

10x Taq buffer

Taq polymerase

dNTPs (10 mM)

* Tagging primer mix

** PNAs (mix of mPNA and pPNA each at $50 \mu \mathrm{M}$ )

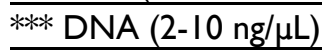

Water

* tagging primer mix is an equimolar mix of tagging

primers with each at a partial concentration of I.25 $\mu \mathrm{M}$

** PNA was used to block chloroplast and mitochondrial amplification in reactions involving the $\mathrm{V} 4$ or $\mathrm{V} 3 \mathrm{~V} 4$ region of I6S rDNA. For other reactions, it is not helpful and is omitted.

*** not part of master mix

Each well received $20 \mu \mathrm{L}$ of master mix and $5 \mu \mathrm{L}$ of DNA (around $50 \mathrm{ng}$ ). Completed reactions were thoroughly mixed on a plate vortex and placed into a preheated thermocycler. We used the following standard cycling conditions:

1) $94^{\circ} \mathrm{C}$ for 2 min. Denature

2) $78^{\circ} \mathrm{C}$ for $10 \mathrm{sec}$. PNA annealing

3) $58^{\circ} \mathrm{C}$ for $15 \mathrm{sec}$. Primer annealing

4) $55^{\circ} \mathrm{C}$ for $15 \mathrm{sec}$. Primer annealing

5) $72^{\circ} \mathrm{C}$ for I min. Extension

6) GO TO STEP I for I additional cycle

7) $16^{\circ} \mathrm{C}$ forever Hold
For $25 \mu L$

$2.5 \mu \mathrm{L}$

$0.2 \mu \mathrm{L}$

$0.5 \mu \mathrm{L}$

$1.25 \mu \mathrm{L}$

$0.375 \mu \mathrm{L}$

$5 \mu \mathrm{L}$

(to $25 \mu \mathrm{L}$ ) 
The tagging reaction was cleaned with Solid Phase Reversible Immobilization (SPRI) beads $^{36}$. All ITS amplicons were cleaned with a I.I:I ratio of SPRI beads to DNA, or $27.5 \mu \mathrm{L}$ beads mixed in $25 \mu \mathrm{L}$ of tagged template. After securing beads and DNA to a magnet and removing the supernatant containing primers and small fragments, beads were washed twice with $80 \%$ ethanol, air dried briefly, and eluted in $17 \mu \mathrm{L}$ of water. For primer sequences, see Supplementary Information and Supplementary Table I.

\section{Exponential reaction}

$15 \mu \mathrm{L}$ of the tagged DNA from step one was used as template for the exponential reaction. To reduce errors during the exponential phase, we used the proof-reading enzyme Q5 from NEB, with its included buffer. We prepared reactions in $25 \mu \mathrm{L}$ for technical tests with replicated samples. For samples prepared without sequenced replicates, we prepared most in triplicate reactions in which a $40 \mu \mathrm{L}$ mix was split into 3 parallel reactions of $\sim 13 \mu \mathrm{L}$ prior to PCR to reduce bias, although this is likely unnecessary ${ }^{69}$.

\begin{tabular}{lll} 
Master Mix & For $25 \mu \mathrm{L}$ & For $\mathbf{4 0} \mu \mathrm{L}$ \\
\hline 5x Q5 buffer & $5 \mu \mathrm{L}$ & $8 \mu \mathrm{L}$ \\
\hline Q5 polymerase & $0.25 \mu \mathrm{L}$ & $0.4 \mu \mathrm{L}$ \\
\hline dNTPs $(10 \mathrm{mM})$ & $0.5 \mu \mathrm{L}$ & $0.8 \mu \mathrm{L}$ \\
\hline 100 $\mu \mathrm{M}$ F universal PCR primer & $0.0625 \mu \mathrm{L}$ & $0.1 \mu \mathrm{L}$ \\
\hline * PNAs (mix of mPNA and pPNA each at $50 \mu \mathrm{M})$ & $0.375 \mu \mathrm{L}$ & $0.6 \mu \mathrm{L}$ \\
\hline ** $5 \mu \mathrm{M}$ reverse barcoded primer & $1.25 \mu \mathrm{L}$ & $2 \mu \mathrm{L}$ \\
\hline ** DNA (from previous reaction) & $15 \mu \mathrm{L}$ & $15 \mu \mathrm{L}$ \\
\hline Water & (to $25 \mu \mathrm{L})$ & (to $40 \mu \mathrm{L})$
\end{tabular}

* PNA was used to block chloroplast and mitochondrial amplification in reactions involving the $\mathrm{V} 4$ or $\mathrm{V} 3 \mathrm{~V} 4$ region of I6S rDNA. For other reactions, it is not helpful and is omitted.

** not part of master mix

We first distributed $8.75 \mu \mathrm{L}$ (or $23 \mu \mathrm{L}$ for $40 \mu \mathrm{L}$ mixes) of master mix to each well. We then added $15 \mu \mathrm{L}$ of the DNA from the tagging reaction and I. $25 \mu \mathrm{L}$ (or $2 \mu \mathrm{L}$ for $40 \mu \mathrm{L}$ mixes) of $5 \mu \mathrm{M}$ barcoded reverse primer. For the $40 \mu \mathrm{L}$ mixes, I $3 \mu \mathrm{L}$ was pipetted into two new PCR wells. The PCR reactions were placed into a hot thermocycler and cycled with the following standard conditions: 
1) $94^{\circ} \mathrm{C}$ for $2 \mathrm{~min}$. Denature

2) $94^{\circ} \mathrm{C}$ for $20 \mathrm{sec}$. Denature

3) $78^{\circ} \mathrm{C}$ for $5 \mathrm{sec}$. PNA annealing

4) $60^{\circ} \mathrm{C}$ for $30 \mathrm{sec}$. Primer annealing

5) $72^{\circ} \mathrm{C}$ for $45 \mathrm{sec}$. Extension

6) GO TO STEP 2 for 29 additional cycles

7) $16^{\circ} \mathrm{C}$ forever Hold

Following PCR, sets of three $\mathrm{I} 3 \mu \mathrm{L}$ reactions were recombined to $40 \mu \mathrm{L}$. For primer sequences, see Supplementary Information and Supplementary Table I.

\section{Library quality control and pooling}

For visualization, $5 \mu \mathrm{L}$ of PCR product was mixed with $3 \mu \mathrm{L}$ of $6 x$ loading dye and all 8 $\mu \mathrm{L}$ loaded on a $2 \%$ agarose gel and stained with ethidium bromide. The remaining PCR products were cleaned with a SPRI-to-DNA ratio of I.I:I.0 (v/v). The DNA concentrations in the cleaned products were measured with PicoGreen (Invitrogen, Carlsbad, CA, USA) and samples were pooled at equimolar total DNA ratios. We note that because host and microbial fractions are independently visible on the gel, it would also be possible to measure the quantity of microbial products with image analysis software such as Image ${ }^{70}$ and pool at equimolar microbial ratios.

The pooled library was diluted to $\sim \mathrm{ng} / \mathrm{uL}$ and run on a Bioanalyzer High Sensitivity DNA chip (Agilent, Santa Clara, CA, USA) to check library purity and to estimate the expected ratio of host to microbial amplicons in the sample.

\section{Pre-sequencing adjustment of host : microbe Ratio}

To adjust the host-to-microbe ratio in the "synthetic template panel" and "cycle number test" prior to sequencing on a HiSeq3000 instrument (Illumina, San Diego, CA, USA), four reference samples were first made by rebarcoding the original pooled library (Supplementary Figures 9 and 10). To accomplish this, $\sim 5 \mathrm{ng}$ of of the pooled library was used in a $30 \mu \mathrm{L}$ PCR reaction as follows:

Master Mix

\begin{tabular}{ll}
\hline $5 \times \mathrm{Q} 5$ buffer & $6 \mu \mathrm{L}$ \\
\hline Q5 polymerase & $0.3 \mu \mathrm{L}$ \\
\hline dNTPs $(10 \mathrm{mM})$ & $0.6 \mu \mathrm{L}$ \\
\hline $100 \mu \mathrm{M}$ F universal PCR primer & $0.075 \mu \mathrm{L}$ \\
\hline ** $5 \mu \mathrm{M}$ reverse barcoded primer & $1.5 \mu \mathrm{L}$ \\
\hline ** $5 \mathrm{ng}$ original pooled library & $5 \mu \mathrm{L}$ \\
\hline Water & $16.45 \mu \mathrm{L}$
\end{tabular}

** not part of master mix

For $30 \mu \mathrm{L}$ 
After distributing $23.5 \mu \mathrm{L}$ of master mix to each well, $5 \mu \mathrm{L}$ of the diluted original library was added to each well ( $5 \mathrm{ng}$ total), along with $\mathrm{I.5} \mu \mathrm{L}$ of $5 \mu \mathrm{M}$ barcoded reverse primer. Just prior to placing the reactions in the thermocycler, a $5 \mu \mathrm{L}$ pre-PCR aliquot was removed from each one and kept on ice to preserve the pre-PCR concentrations. The remaining $25 \mu \mathrm{L}$ reaction was placed into a preheated thermocycler and run for 8 cycles, using the following cycling conditions:

1) $94^{\circ} \mathrm{C}$ for $2 \mathrm{~min}$. Denature

2) $94^{\circ} \mathrm{C}$ for $30 \mathrm{sec}$. Denature

3) $78^{\circ} \mathrm{C}$ for $5 \mathrm{sec}$. PNA annealing

4) $60^{\circ} \mathrm{C}$ for I min. Primer annealing

5) $72^{\circ} \mathrm{C}$ for I min. Extension

6) GO TO STEP 2 for 7 additional cycles

7) $16^{\circ} \mathrm{C}$ forever Hold

Following PCR, the pre-PCR aliquots were run alongside $5 \mu \mathrm{L}$ of post-PCR product on a $2 \%$ gel to confirm successful amplification of the reference libraries (Supplementary Figure 10). The remaining $20 \mu \mathrm{L}$ of PCR reactions were then cleaned with SPRI beads (I.5: $1.0[\mathrm{v} / \mathrm{v}])$ and set aside. A large aliquot of the original library (approximately $50 \mathrm{ng}$ ) was also run on a $2 \%$ gel to separate the host and microbe bands for individual purification. The bands were cut out of the gel and each band was put into a separate Econospin spin column (Epoch Life Sciences, Missouri City, TX, USA) without any other liquids or binding buffer. The gel slices were centrifuged at maximum speed to force the liquid containing the DNA into the bottom chamber, leaving the dried gel on top. The eluted DNA was cleaned with SPRI beads at I.5 : 1.0 $(\mathrm{v} / \mathrm{v})$ and eluted in EB.

The purified pooled host library fraction, pooled microbe library fraction, and each of the four reference libraries were quantified with Picogreen and the molarity of each was estimated. The pools were then mixed together, targeting host molarity at $5 \%$ of the total and each reference library at $1 \%$ of the total.

\section{Illumina sequencing}

Pooled and quality-checked sequencing libraries were cleaned of all remaining dimers and off-target fragments using a BluePippin (Sage Science, Beverly, MA, USA) set to a broad range of 280 to $720 \mathrm{bp}$. The libraries were then diluted for Illumina sequencing following manufacturers' protocols. Libraries were first diluted to $2.5-2.8 \mathrm{nM}$ in elution buffer $(E B, 10$ $\mathrm{mM}$ Tris $\mathrm{pH} 8.0)$ and spiked into a compatible lane of the HiSeq3000 instrument $(2 \times 150 \mathrm{bp}$ paired end reads) to occupy $2-3 \%$ of the lane. Samples were sequenced across four total lanes (Supplementary Table I) 


\section{Sequence processing}

The sequences were demultiplexed first by the 9 bp barcode on the PCR primers (Supplementary Table I), of which there are 96, not allowing for any mismatches. In some cases in which two samples differed in both their host and microbe primer sets, we amplified both samples with the same 9 bp barcode to increase multiplexing; such samples were further demultiplexed using regular expressions for the forward primer and reverse primer sequences. Following demultiplexing, all samples were filtered to remove sequences with any mismatches to the expected primers. With HiSeq3000 I50 bp read lengths, overlap of read I and read 2 was not possible for our amplicons, and therefore only read I was processed further.

All primer sequences were removed. Additional quality filtering, removal of chimeric sequences, preparation and Amplicon Sequence Variant (ASV) tables, and taxonomic assignment were done with a combination of VSEARCH ${ }^{71}$ and USEARCHIO ${ }^{39}$. ASVs were prepared as 'zero-radius OTUs' (zOTUs) ${ }^{39}$. The I6S rDNA taxonomy was classified based on the RDP training set $\mathrm{vl} 6$ ( $13 \mathrm{~K}$ seqs.) ${ }^{72}$, and ITSI taxonomy of the top 10 most abundant fungal ASVs was classified manually using the UNITE database ${ }^{73}$ (https://unite.ut.ee/). To reduce memory usage, data from the five lanes was processed into four independent ASV tables (Supplementary Data), as described in the sample metadata (Supplementary Table I).

ASV tables were analyzed statistically and graphically using custom scripts in $R^{74}$, particularly with the help of packages "ggplot2"75 and "reshape2"76. Custom scripts are available on GitHub at (https://github.com/derekLSI/hamPCR).

\section{Samples}

\section{Synthetic titration panel}

Seeds from the Arabidopsis thaliana accession Col-0 were surface sterilized by immersion for I minute in $70 \%$ ethanol with $0.1 \%$ Triton X-100, soaking in 10\% household bleach for 12 minutes, and washing three times with sterile water. Seeds were germinated axenically on $1 / 2$ strength MS media with MES, and about $2 \mathrm{~g}$ of seedlings were harvested after 10 days. DNA was extracted in the sterile hood as in ${ }^{12}$ and diluted to $10 \mathrm{ng} / \mu \mathrm{L}$ in elution buffer (I0 mM Tris $\mathrm{pH} 8.0$, hereafter EB). Pure E. coli and Sphingomonas sp. cultures were likewise grown with LB liquid and solid media respectively, and DNA was extracted using a bead beating protocol $^{\prime 2}$. E. coli DNA was used separately, or alternatively pooled with the mixed Sphingomonas DNA, and diluted to $10 \mathrm{ng} / \mu \mathrm{L}$. The plant DNA and microbial DNA were then combined according to the following table: 


\begin{tabular}{|c|c|c|c|c|c|c|c|c|c|c|c|c|c|c|}
\hline & IOOE & 100 & 24 & 20 & 16 & 8 & 4 & 2 & I & 0.5 & 0.25 & 100P & 100P & blank \\
\hline$\mu \mathrm{L}$ A .thaliana DNA (10 ng/ $\mu \mathrm{L})$ & & & 760 & 800 & 840 & 920 & 960 & 980 & 990 & 995 & 997.5 & 1000 & 1000 & \\
\hline$\overline{\mu L}$ mixed bacterial DNA (10ng/ $\mu \mathrm{L})$ & & 1000 & 240 & 200 & 160 & 80 & 40 & 20 & 10 & 5 & 2.5 & & & \\
\hline 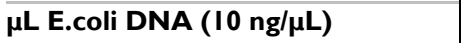 & 1000 & & & & & & & & & & & & & \\
\hline$\mu \mathrm{L}$ water & & & & & & & & & & & & & & 1000 \\
\hline
\end{tabular}

V4 tagging was performed with 5I5_FI_G-46603 and 799_RI_G-4660I (V4 I6S rDNA) and At.Gl_FI_G-46602 and At.GI_R502bp_G-466I4 (A. thaliana Gl). Each exponential PCR reaction was completed in a single reaction of $25 \mu \mathrm{L}$.

\section{Synthetic equimolar plasmid template}

The ITSI region from Agaricus bisporus, a fragment of the $G I$ gene from $A$. thaliana Col-0 accession, the I6S rRNA gene from Pto DC3000, and the ITSI region from $H$. arabidopsidis were PCR amplified individually, combined into one fragment via overlap extension PCR, and cloned into PGEM®-T Easy (Promega, Madison, WI, USA). The sequences of these templates can be found in Supplementary Information.

\section{Wild A. thaliana samples}

DNA from chosen samples previously analyzed by conventional I6S rDNA-only sequencing of the V4 region and shotgun metagenomics ${ }^{12}$ was reused, chosen to capture a wide range of realistic bacterial loads. The samples were individually assayed with hamPCR using $5 \mu \mathrm{L}$ DNA template (approximately $50 \mathrm{ng}$ ). V4 tagging was performed with 5I5_FI_G-46603 and 806_RI_G-4663I (V4 I6S rDNA) and At.GI_FI_G-46602 and At.GI_R502bp_G-466I4 (502 bp $A$. thaliana GI). These were the only V4 samples tagged with the 806R primer instead of the nearby $799 \mathrm{R}$ primer, and it was used to enable direct comparison to the dataset in ${ }^{12}$. V3V4 tagging was performed with 34I_FI_G-46605 and 799_RI_G-4660I (V3V4 I6S rDNA) and At.GI_FI_G-46602 and At.GI_R502bp_G-466I4 (502 bp A. thaliana GI). V5V6V7 tagging was performed with 799_FI_G-46628 and II92_RI_G-46629 (V5V6V7 I6S rDNA) and At.GI_FI_G-46602 and At.GI_R466bp_G-46652 (466 bp A. thaliana GI). Each exponential PCR reaction was completed in a single reaction of $25 \mu \mathrm{L}$; each sample was replicated three times.

\section{Wild A. thaliana mixed sample}

DNA from samples previously analyzed by conventional I6S rDNA-only sequencing of the V4 region and shotgun metagenomics ${ }^{12}$ were pooled to prepare a single abundant mixed sample to be used repeatedly for technical tests. 


\section{Hyaloperonospora arabidopsidis and Pseudomonas syringae pv. tomato DC3000 co-infection}

Both wildtype $A$. thaliana seedlings in the Col-0 genetic background and enhanced disease susceptibility I mutants in the Ws-0 genetic background (eds/-I) were grown from surface-sterilized seeds. Seedlings were raised in ED73 potting mix (Einheitserdewerke, Sinntal-Altengronau, Germany) in $5 \mathrm{~cm}$ pots for 10 days under short-day conditions (8 hours light, 16 hours dark). Each pot contained 4 to 5 seedlings, and for each genotype, four pots were used for each infection condition. Plants were treated with either $10 \mathrm{mM} \mathrm{MgCl}{ }_{2}($ buffer only), $H$. arabidopsidis (Hpa) isolate $466-\mathrm{I}$ alone $\left(5 \times 10^{4}\right.$ spores / $\left.\mathrm{mL}\right)$, or Hpa 466-I with $P$. syringae pv. tomato (Pto) DC3000 (OD600 $=0.25$, a gift from El Kasmi lab, University of Tübingen).

The infected plants were grown at $16^{\circ} \mathrm{C}$ for 8 days (10 hours light, 14 hours dark) and harvested by pooling all seedlings in each pot into a sterile pre-weighed tube, which was again weighed to find the mass of the seedlings. Three $5 \mathrm{~mm}$ glass balls and $300 \mu \mathrm{L} 10 \mathrm{mM} \mathrm{MgCl}$ were added to each tube and the plant cells were lysed at a speed of $4.0 \mathrm{~m} / \mathrm{s}$ for 20 seconds in a FastPrep-24 ${ }^{\mathrm{TM}}$ Instrument (MP Biomedicals, Illkirch-Graffenstaden, France) to release the live bacteria from the leaves. From the pure lysate, $20 \mu \mathrm{L}$ was used for a serial log dilution series, and $5 \mu \mathrm{L}$ of each dilution was plated on LB agar supplemented with $100 \mu \mathrm{g} / \mathrm{mL}$ rifampicin. Colony forming units (CFUs) were counted after 2 days of incubation at $28^{\circ} \mathrm{C}$. The remaining $280 \mu \mathrm{L}$ of lysate were combined with $520 \mu \mathrm{L}$ DNA lysis buffer, $0.5 \mathrm{~mL}$ of I mm garnet sharp particles (BioSpec, Bartlesville, OK, USA). $60 \mu \mathrm{L}$ of 20\% SDS was added to make a final SDS concentration of $1.5 \%$, and DNA was extracted using a bead beating protocol ${ }^{12}$. The number of Hpa sporangiophores was too high to be accurately quantified by visual counting.

The DNA preps were individually assayed with hamPCR using $5 \mu \mathrm{L}$ DNA template (approximately $30 \mathrm{ng}$ ); tagging was performed with three primer sets: Ha.Actin_FI_G-467I6 and Ha.Actin_RI_G-467I7 (Hpa Actin), At.Gl_FI_G-46602 and At.GI_R502bp_G-466I4 (502 bp $A$. thaliana GI), and 5I5_FI_G-46603 and 799_RI_G-4660I (V4 I6S rDNA). Each exponential PCR reaction was completed in three parallel reactions of $13 \mu \mathrm{L}$, which were recombined prior to sequencing.

\section{Titration with plant DNA infected with $\boldsymbol{H}$. arabidopsidis}

A titration panel was made combining different amounts of DNA from uninfected plants (eds $/$ - I treated only with $10 \mathrm{mM} \mathrm{MgCl}_{2}$ ) and DNA from $H$ pa-infected plants (eds/-I infected with $\mathrm{Hpa}$ as described above). Infected and uninfected pools were each diluted to $6 \mathrm{ng} / \mu \mathrm{L}$, and combined in 0:7, I:6, 2:5, 3:4, 4:3, 5:2, 6:1, and 7:0 ratios. These were tagged using the same three primer sets described above for Hpa actin, A. thaliana Gl, and V4 I6S rDNA above. Each 
exponential PCR reaction was completed in a single reaction of $25 \mu \mathrm{L}$; hamPCR was replicated on the titration three times.

\section{Capsicum annuum infections with Xanthomonas}

Leaf infiltration log series: Using pressure infiltration with a blunt-end syringe, $C$. annuum cultivar Early Calwonder (ECW) leaves were inoculated with Xanthomonas euvesicatoria $(X e)$. Xe strain $85-10^{53}$ was resuspended in $10 \mathrm{mM} \mathrm{MgCl}_{2}$ to final concentration of $10^{8} \mathrm{CFU} / \mathrm{mL}\left(\mathrm{OD}_{600}=0.4\right)$ and further diluted to $10^{7}, 10^{6}, 10^{5}$ and $10^{4} \mathrm{CFU} / \mathrm{mL}$. Upon infiltration, 5 leaf discs (7 mm diameter) were punched from each leaf per sample and placed in a $2 \mathrm{~mL}$ round-bottom tube with two SiLibeads (type ZY-S 2.7-3.3 mm, Sigmund Lindner GmbH, Warmensteinach, Germany) and $300 \mu \mathrm{L} 10 \mathrm{mM} \mathrm{MgCl}$. The samples were ground by bead beating for $25 \mathrm{sec}$ at $25 \mathrm{~Hz}$ using a Tissue Lyser II machine (Qiagen, Hilden, Germany). For CFU-based bacterial enumeration, $30 \mu \mathrm{L}$ of the lysate or $30 \mu \mathrm{L}$ of serial dilutions were plated on NYG medium $(0.5 \%$ peptone, $0.3 \%$ yeast extract, $0.2 \%$ glycerol and $1.5 \%$ agar 77 containing rifampicin $(100 \mu \mathrm{g} / \mathrm{ml})$. Xe bacteria were counted 3 days post incubation at $28^{\circ} \mathrm{C}$. The remaining $250 \mu \mathrm{L}$ of lysate was combined with $600 \mu \mathrm{L}$ of DNA lysis buffer containing $2.1 \%$ SDS (for a $1.5 \%$ final SDS concentration) and transferred to screw cap tubes filled with I mm garnet sharp particles, for a bead-beating DNA prep as previously described ${ }^{12}$.

Growth curve: $X e$ strain $85-10$, resuspended in $10 \mathrm{mM} \mathrm{MgCl}$ to a final concentration of $10^{4} \mathrm{CFU} / \mathrm{mL}$, was infiltrated via a blunt end syringe into 6 C. annuum (ECW) leaves of 6 different plants. Upon 0, 2, 4, 7, 9 and II dpi (days post inoculation) 4 leaf discs (7 mm diameter) from each inoculated leaf were harvested and bacterial numbers were determined as described above. $250 \mu \mathrm{L}$ of leaf lysates were used for a bead-beating DNA prep as described for all other samples above.

Each hamPCR template tagging reaction used 5-10 $\mu \mathrm{L}$ template (approximately $50 \mathrm{ng}$ each); tagging was performed with primers 5I5_F3_G-46694 and 799_RI_G-4660I (V4 I6S rDNA), and Ca.GI_FI_G-46626 and Ca.GI_RI_G-46627 (C. annuum GI). Each exponential $\mathrm{PCR}$ reaction was completed in three parallel reactions of $\mathrm{I} 3 \mu \mathrm{L}$, which were recombined prior to sequencing.

\section{Pristionchus pacificus titration panel}

Pristionchus pacificus strain PS312 $2^{78}$ was grown on nematode growth media (NGM) plates supporting a bacterial lawn of either pure $E$. coli OP50 or alternatively a mix of $E$. coli OP50, Pto DC3000, and Lysinibacillus xylanilyticus (a strain isolated from wild $P$. pacificus). The worms were washed extensively with PBS buffer to remove epidermally-attached bacteria, and DNA was prepared from whole worms using the same bead beating protocol as described for A. thaliana ${ }^{12}$. Worm DNA from the pure culture and the mixed culture were each diluted to 6 $\mathrm{ng} / \mu \mathrm{L}$, and combined in 0:7, I:6, 2:5, 3:4, 4:3, 5:2, 6:1, and 7:0 ratios to create a titration panel. 
Each hamPCR template ( $5 \mu \mathrm{L}$ template or $30 \mathrm{ng}$ total) was used to perform the tagging reaction, using primers 799FI_G-46628 and II92RI_G-46629 (V5V6V7 I6S rDNA), and Pp_csq-I_FI_G-4669I and Pp_csq-I_RI_G-46692 (P. pacificus csq-I). Each exponential PCR reaction was completed in a single reaction of $25 \mu \mathrm{L}$; the titration was replicated three times.

\section{Triticum aestivum titration panel}

Triticum aestivum (wheat) seeds (Rapunzel Naturkost, Legau, Germany) were surface-sterilized by immersion in $70 \%$ ethanol and $0.1 \%$ Triton X-100 for I minute, soaking for 15 minutes in 10\% household bleach, and finally washing three times in sterile autoclaved water. Axenic plants were grown on I\% agar supplemented with I/2 strength MS medium buffered with MES. About I $g$ of sterile leaf tissue was harvested after 10 days, and DNA was extracted in the sterile hood as described in ref. ${ }^{12}$. Roots that had been spontaneously colonized by microbes were obtained by growing by transplanting germinated seeds outdoors into potting soil. Roots were harvested from approximately 4-week old plants and surface-sterilized by immersion in 10\% household bleach with $0.1 \%$ Triton X-100 for 5 minutes, followed by 3 washes with sterile water. Axenic leaf DNA and spontaneously-colonized root DNA were each diluted to $60 \mathrm{ng} / \mu \mathrm{L}$ and combined in 0:7, I:6, 2:5, 3:4, 4:3, 5:2, 6:1, and 7:0 ratios to create a titration panel of eight samples. Each hamPCR tagging reaction used $3 \mu \mathrm{L}$ ( $180 \mathrm{ng}$ ) template; fungal ITSI tagging was performed with primers ITSI_FI_G-46622 and ITS2_RI_G-46623 (ITSI rDNA), and PolAI_FI_G-46750 and PolAI_RI_G-4675I (T. aestivum RNA polymerase I gene, PolA/). Bacterial I6S rDNA tagging was performed with the same PolAl primers and with 5I5_FI_G-46603 and 799_RI_G-4660I (V4 I6S rDNA). To make an additional ITSI library enriched for ITSI amplicons, the ITSI primer pair concentration was increased by a factor of $\mathrm{I} .33$ and the PolAI primer pair concentration was decreased by a factor of 0.66 , giving a 2:I ratio instead of the standard I:I ratio, and the tagged products were amplified with 7 tagging and 25 PCR cycles instead of the standard 2 tagging and 30 PCR cycles.

\section{Zea mays field samples}

Samples of leaves from mature Zea mays (maize) genotype B73 were harvested by standard hole punch from a field side in Tübingen. Permission to punch the leaves was graciously provided by Dr. Marja Timmermans (University of Tübingen). Each sample comprised 5 leaf discs, which were immediately shaken in $1 \mathrm{~mL}$ of sterile water in a screw cap tube to remove dust from the field. The water was removed by pipetting and the leaf discs were snap frozen in liquid nitrogen and taken back to the lab for processing. DNA was extracted with the bead beating protocol described above, with the difference that prior to addition of lysis buffer and garnet rocks, the deep frozen leaf discs were pre-ground with 3 metal ball bearings at a speed of $5.0 \mathrm{~m} / \mathrm{s}$ in a FastPrep-24 ${ }^{\text {TM }}$ instrument. We found this pre-grind was helpful to break down the fibrous maize leaf tissue. Prior to adding garnet rocks and lysis buffer, the metal balls 
were removed by magnet, as metal balls can crack the tubes at the speed of $6.0 \mathrm{~m} / \mathrm{s}$ used for the primary DNA extraction. Each hamPCR tagging reaction used $10 \mu \mathrm{L}(\sim 120 \mathrm{ng})$ template; Bacterial I6S rDNA was tagged with one of the forward primers 5I5F_bcGA_G-47I88, 5I5F_bcTC_G-47I89, 5I5F_bcAG_G-47190, or 5I5_F3_G-46694 paired with the reverse primer 799_RI_G-4660I (V4 I6S rDNA). Maize LUMINIDEPENDENS (LD) was tagged with one of the forward LDPI primers Zm_LD_bcGA_G-47I84, Zm_LD_bcTC_G-47I85, Zm_LD_bcAG_G-47I86, or Zm_LD_bcCT_G-47I87 paired with the $L D$ reverse primer Zm_LD_R_G-47I58. the standard 2 tagging and 30 PCR cycles. Two tagging cycles were paired with 30 exponential cycles. To reduce host representation in the final library from the original $\sim 75 \%$ to approximately $40 \%$, we used the gel remixing technique described in Figure 3.

\section{Test of tagging step cycle numbers}

As templates, we used a pool of mixed wild $A$. thaliana leaf DNA ( $50 \mathrm{ng} /$ reaction) and the "synthetic equimolar plasmid template" ( $0.05 \mathrm{ng} /$ reaction). For the wild $A$. thaliana leaf DNA, we tested V4 I6S rDNA primers alone in the tagging step vs. hamPCR with V4 I6S rDNA primers plus primers for the host $G$ gene. For the "synthetic equimolar plasmid template", we used only hamPCR. Specifically, we used 5I5_FI_G-46603 and 799_RI_G-4660I (V4 I6S rDNA) and At.GI_FI_G-46602 and At.GI_R502bp_G-466I4 (502 bp Gl gene).

We applied hamPCR for 2, 3, 4, 5, 6, 7, 8, 9, or 10 tagging cycles, paired with $30,29,28$, $27,26,25,24,23$, or 22 PCR cycles, respectively. All tagging and PCR reactions were started together, and fewer tagging cycles than 10, or fewer PCR cycles than 30, were achieved by taking PCR tubes out of the thermocycler at the end of the appropriate extension steps and placing them on ice.

\section{Tests of template concentrations}

A panel of 8 concentrations of wild $A$. thaliana leaf DNA was prepared, ranging from 5 to $500 \mathrm{ng}$ per reaction. Primers for the wild $A$. thaliana leaf DNA were 5I5_FI_G-46603 and 799_RI_G-4660I (V4 I6S rDNA) and At.GI_FI_G-46602 and At.GI_R502bp_G-466I4 (502 bp GIGANTEA gene), with both primer pairs in equal ratio.

\section{Quantitative real time PCR on C. annuum samples}

A primer set targeting the gene for the type III effector XopQ of pathogenic Xanthomonas was used to measure abundance of $X e$ 85-1054. For $C$. annuum, primers targeting the UBI-3 gene encoding a ubiquitin-conjugating protein were used ${ }^{55}$. Two reagent mastermixes were prepared, one for each primer set, to help improve primer dose consistency. Each sample was amplified using three $10 \mu \mathrm{L}$ technical replicates per primer set that were 
averaged for analysis. Each $10 \mu \mathrm{L}$ reaction included $2.5 \mu \mathrm{L}$ of DNA, to which was added, as a mastermix, $5 \mu \mathrm{L}$ SYBR尺 Green PCR Master Mix (Life Technologies, Carlsbad, California), I.5 $\mu \mathrm{L}$ water, $0.5 \mu \mathrm{L}$ of $5 \mu \mathrm{M}$ forward primer, and $0.5 \mu \mathrm{L}$ of $5 \mu \mathrm{M}$ reverse primer. qPCR was performed on a BioRad CFX384 Real-time System and analyzed with the CFX Manager Software. The following conditions were used for the amplification of both target genes:

1) $94^{\circ} \mathrm{C}$ for $5 \mathrm{~min}$. Denature

2) $94^{\circ} \mathrm{C}$ for $30 \mathrm{sec}$. Denature

3) $55^{\circ} \mathrm{C}$ for $30 \mathrm{sec}$. Annealing

4) $68^{\circ} \mathrm{C}$ for $45 \mathrm{sec}$. Extension

5) Image fluorescence

6) GO TO STEP 2 for 39 additional cycles

The ratio of microbial to host DNA was initially calculated as $2^{\wedge}(-$ mean $x o p Q C q$ value) I 2^(-mean UBI-3 Cq value). See alignment to CFU counts below.

\section{Alignment of Xanthomonas qPCR and hamPCR load with CFU counts}

Log10-transformed Xe 85-10 ASV loads from hamPCR were regressed onto $\log 10$-transformed xopQ loads from qPCR (least squares method), and the slope $(m)$ and $y$-intercept $(b)$ of the best-fit line were used to transform and align the qPCR loads to hamPCR loads with the following formula: Load_qPCR hamPCR-aligned $=m \times$ Load_qPCR $+b$. Next, $\log 10$-transformed CFU counts were regressed onto the log 10-transformed hamPCR loads, and the slope and $y$-intercept of the resulting best-fit line were used similarly to align both Load_qPCR hamPCR-aligned and hamPCR loads to the CFU counts.

\section{Correlation networks}

Pearson correlation matrices for relative abundance and microbial load data were created in $\mathrm{R}^{74}$ using the "stats" package. The SparCC ${ }^{56}$ correlation matrix was created in $\mathrm{R}$ using the implementation in the "SpiecEasi" package ${ }^{79}$. Networks were visualized with the package "qgraph"80. Custom scripts are available on GitHub (https://github.com/derekLSI/hamPCR). 


\section{Data availability}

All data in this manuscript has been deposited in the European Nucleotide Archive (ENA). It can be accessed under the project number PRJEB38287. At https://www.ebi.ac.uk/ena.

\section{Author contributions}

DL and PP planned the study. DL led the data analysis. DL and PP performed all DNA extractions and prepared all hamPCR libraries for sequencing. PP constructed the synthetic plasmid template. AS and TL planned, and AS conducted, the pepper and Xanthomonas infections. DL and GS planned and conducted the Hpa and Pto DC3000 co-infection of $A$. thaliana. WL grew and harvested the colonized $P$. pacificus samples. DL, PP, and DW wrote the manuscript.

\section{Acknowledgements}

We thank Lei Li and Fiona Beitel for discussions and suggestions regarding PCR and qPCR, Talia Karasov for suggestions on presentation, Katrin Fritschi and Heike Budde for technical help with sequencing, Ilja Bezrukov for assistance with demultiplexing, and Dor Russ, Jeff Dangl, and Benjamin Schwessinger for supportive discussions and comments on the manuscript. Thanks to Victor Schmidt, Nick Youngblut, Lei Li, and Hua Wang for giving us space in their HiSeq 3000 lanes. Thanks to Marja Timmermans for access to field maize plants. Supported by a Human Frontiers Science Program (HFSP) Long-Term Fellowship (LT000565/20I5-L to DL), the DFG through Priority Program SPP DECRyPT, and the Max Planck Society (DW). 


\section{Competing interests}

The authors declare no competing interests.

\section{References}

I. Tsilimigras, M. C. B. \& Fodor, A. A. Compositional data analysis of the microbiome: fundamentals, tools, and challenges. Ann. Epidemiol. 26, 330-335 (2016).

2. Gloor, G. B., Macklaim, J. M., Pawlowsky-Glahn, V. \& Egozcue, J. J. Microbiome Datasets Are Compositional: And This Is Not Optional. Front. Microbiol. 8, 2224 (2017).

3. Barlow, J. T., Bogatyrev, S. R. \& Ismagilov, R. F. A quantitative sequencing framework for absolute abundance measurements of mucosal and lumenal microbial communities. Nat. Commun. I I, 2590 (2020).

4. Morton, J. T. et al. Establishing microbial composition measurement standards with reference frames. Nat. Commun. I0, 2719 (2019).

5. Vandeputte, D. et al. Quantitative microbiome profiling links gut community variation to microbial load. Nature 55 I, 507-5I I (20I7).

6. Humphrey, P. T. \& Whiteman, N. K. Insect herbivory reshapes a native leaf microbiome. Nature Ecology \& Evolution I-9 (2020).

7. Props, R. et al. Absolute quantification of microbial taxon abundances. ISME J. I I, 584 (20I6).

8. Tkacz, A., Hortala, M. \& Poole, P. S. Absolute quantitation of microbiota abundance in environmental samples. Microbiome 6, II0 (2018).

9. Smets, W. et al. A method for simultaneous measurement of soil bacterial abundances and community composition via I6S rRNA gene sequencing. Soil Biol. Biochem. 96, I45-I5I (20I6).

10. Stämmler, F. et al. Adjusting microbiome profiles for differences in microbial load by spike-in bacteria. Microbiome 4, 28 (2016).

II. Niu, B., Paulson, J. N., Zheng, X. \& Kolter, R. Simplified and representative bacterial community of maize roots. Proc. Natl. Acad. Sci. U. S. A. I I 4, E2450-E2459 (20I7).

12. Regalado, J. et al. Combining whole-genome shotgun sequencing and rRNA gene amplicon analyses to improve detection of microbe-microbe interaction networks in plant leaves. ISME J. 14 , 2II6-2I 30 (2020).

13. Ogier, J.-C., Pagès, S., Frayssinet, M. \& Gaudriault, S. Entomopathogenic nematode-associated microbiota: from monoxenic paradigm to pathobiome. Microbiome 8, 25 (2020).

14. Parker, E. S., Newton, I. L. G. \& Moczek, A. P. (My Microbiome) Would Walk 10,000 miles: Maintenance and Turnover of Microbial Communities in Introduced Dung Beetles. Microb. Ecol. (2020) doi:I0.1007/s00248-020-0I5 I4-9.

15. Gendrin, M. et al. Antibiotics in ingested human blood affect the mosquito microbiota and capacity to transmit malaria. Nat. Commun. 6, 592I (2015).

16. Ellegaard, K. M., Suenami, S., Miyazaki, R. \& Engel, P. Vast Differences in Strain-Level Diversity in the Gut Microbiota of Two Closely Related Honey Bee Species. Curr. Biol. 30, 2520-253 I.e7 (2020).

17. Davies, D. R. DNA contents and cell number in relation to seed size in the genus Vicia. Heredity 39, 153 (1977).

18. Massonnet, C. et al. New insights into the control of endoreduplication: endoreduplication could be driven by organ growth in Arabidopsis leaves. Plant Physiol. 1 57, 2044-2055 (20I I).

19. Karasov, T. L. et al. The relationship between microbial biomass and disease in the Arabidopsis 
thaliana phyllosphere. bioRxiv 8288 I4 (2019) doi: I0.1 I0I/8288I4.

20. Karasov, T. L. et al. Arabidopsis thaliana and Pseudomonas Pathogens Exhibit Stable Associations over Evolutionary Timescales. Cell Host Microbe 24, 168-179.e4 (2018).

21. Lebeis, S. L. et al. Salicylic acid modulates colonization of the root microbiome by specific bacterial taxa. Science 349, 860-864 (20I5).

22. Rabanal, F. A. et al. Unstable Inheritance of $45 \mathrm{~S}$ rRNA Genes in Arabidopsis thaliana. G3 7 , I20I-I 209 (20I7).

23. Anderson, R. G. \& McDowell, J. M. A PCR assay for the quantification of growth of the oomycete pathogen Hyaloperonospora arabidopsidis in Arabidopsis thaliana. Mol. Plant Pathol. I6, 893-898 (20I5).

24. Nadkarni, M. A., Martin, F. E., Jacques, N. A. \& Hunter, N. Determination of bacterial load by real-time PCR using a broad-range (universal) probe and primers set. Microbiology I48, 257-266 (2002).

25. Guo, X. et al. Host-Associated Quantitative Abundance Profiling Reveals the Microbial Load Variation of Root Microbiome. Plant Communications I 00003 (2019).

26. Jian, C., Luukkonen, P., Yki-Järvinen, H., Salonen, A. \& Korpela, K. Quantitative PCR provides a simple and accessible method for quantitative microbiota profiling. PLoS One I 5, e0227285 (2020).

27. Lin, Y., Gifford, S., Ducklow, H., Schofield, O. \& Cassar, N. Towards Quantitative Microbiome Community Profiling Using Internal Standards. Appl. Environ. Microbiol. 85, (2019).

28. Chen, T. et al. A plant genetic network for preventing dysbiosis in the phyllosphere. Nature (2020) doi:10.1038/s41586-020-2185-0.

29. Carlson, C. S. et al. Using synthetic templates to design an unbiased multiplex PCR assay. Nat. Commun. 4, 2680 (20I3).

30. Wen, D. \& Zhang, C. Universal Multiplex PCR: a novel method of simultaneous amplification of multiple DNA fragments. Plant Methods 8, 32 (20I2).

3I. Gohl, D. M. et al. Systematic improvement of amplicon marker gene methods for increased accuracy in microbiome studies. Nat. Biotechnol. 34, 942-949 (2016).

32. Symeonidi, E., Regalado, J., Schwab, R. \& Weigel, D. CRISPR-finder: A high throughput and cost effective method for identifying successfully edited A. thaliana individuals. bioRxiv (2020) doi:10.1 I0I/2020.06.25.171538vI.

33. Lundberg, D. S., Yourstone, S., Mieczkowski, P., Jones, C. D. \& Dangl, J. L. Practical innovations for high-throughput amplicon sequencing. Nat. Methods I0, 999-I002 (20I3).

34. Quince, C., Walker, A. W., Simpson, J. T., Loman, N. J. \& Segata, N. Shotgun metagenomics, from sampling to analysis. Nat. Biotechnol. 35, 833-844 (2017).

35. Kukurba, K. R. \& Montgomery, S. B. RNA Sequencing and Analysis. Cold Spring Harb. Protoc. 20I5, 95I-969 (20I5).

36. Rohland, N. \& Reich, D. Cost-effective, high-throughput DNA sequencing libraries for multiplexed target capture. Genome Res. 22, 939-946 (2012).

37. Duarte, J. M. et al. Identification of shared single copy nuclear genes in Arabidopsis, Populus, Vitis and Oryza and their phylogenetic utility across various taxonomic levels. BMC Evol. Biol. I 0, 6I (20I0).

38. Chelius, M. K. \& Triplett, E. W. The Diversity of Archaea and Bacteria in Association with the Roots of Zea mays L. Microb. Ecol. 4I, 252-263 (200I).

39. Edgar, R. C. Search and clustering orders of magnitude faster than BLAST. Bioinformatics 26, 2460-246I (20I0).

40. Agler, M. T., Mari, A., Dombrowski, N., Hacquard, S. \& Kemen, E. M. New insights in host-associated microbial diversity with broad and accurate taxonomic resolution. bioRxiv 050005 (2016) doi:I0.1 10I/050005. 
4I. Song, L. \& Xie, K. Engineering CRISPR/Cas9 to mitigate abundant host contamination for I6S rRNA gene-based amplicon sequencing. Microbiome 8, 80 (2020).

42. Vet, J. A. et al. Multiplex detection of four pathogenic retroviruses using molecular beacons. Proc. Natl. Acad. Sci. U. S. A. 96, 6394-6399 (1999).

43. Graspeuntner, S., Loeper, N., Künzel, S., Baines, J. F. \& Rupp, J. Selection of validated hypervariable regions is crucial in I6S-based microbiota studies of the female genital tract. Sci. Rep. 8, 9678 (20I8).

44. Kembel, S. W., Wu, M., Eisen, J. A. \& Green, J. L. Incorporating I6S gene copy number information improves estimates of microbial diversity and abundance. PLoS Comput. Biol. 8, el 002743 (20I2).

45. Shuber, A. P., Grondin, V. J. \& Klinger, K. W. A simplified procedure for developing multiplex PCRs. Genome Res. 5, 488-493 (1995).

46. Coates, M. E. \& Beynon, J. L. Hyaloperonospora Arabidopsidis as a pathogen model. Annu. Rev. Phytopathol. 48, 329-345 (2010).

47. Xin, X.-F. \& He, S. Y. Pseudomonas syringae pv. tomato DC3000: a model pathogen for probing disease susceptibility and hormone signaling in plants. Annu. Rev. Phytopathol. 5 I, 473-498 (20I3).

48. Bhandari, D. D. et al. An EDSI heterodimer signalling surface enforces timely reprogramming of immunity genes in Arabidopsis. Nat. Commun. 10, 772 (2019).

49. Huot, B. et al. Dual impact of elevated temperature on plant defence and bacterial virulence in Arabidopsis. Nat. Commun. 8, 1808 (2017).

50. International Wheat Genome Sequencing Consortium (IWGSC) et al. Shifting the limits in wheat research and breeding using a fully annotated reference genome. Science 36 I, (20I8).

5I. Rai, B., Takahashi, H., Kato, K., Sato, Y.-I. \& Nakamura, I. Single-copy nuclear PolAI gene sheds light on the origin of $\mathrm{S}$ genome with relationships to $\mathrm{B}$ and $\mathrm{G}$ genomes of polyploid wheat species. Genet. Resour. Crop Evol. 59, I7I3-1726 (2012).

52. Kim, S. et al. Genome sequence of the hot pepper provides insights into the evolution of pungency in Capsicum species. Nat. Genet. 46, 270-278 (20/4).

53. Thieme, F. et al. Insights into genome plasticity and pathogenicity of the plant pathogenic bacterium Xanthomonas campestris pv. vesicatoria revealed by the complete genome sequence. J. Bacteriol. I 87, 7254-7266 (2005).

54. Doddaraju, P. et al. Reliable and early diagnosis of bacterial blight in pomegranate caused by Xanthomonas axonopodis pv. punicae using sensitive PCR techniques. Sci. Rep. 9, 10097 (2019).

55. Wan, H. et al. Identification of reference genes for reverse transcription quantitative real-time PCR normalization in pepper (Capsicum annuum L.). Biochem. Biophys. Res. Commun. 4I 6, 24-30 $(20 \mathrm{II})$.

56. Friedman, J. \& Alm, E. J. Inferring correlation networks from genomic survey data. PLoS Comput. Biol. 8, el002687 (20I2).

57. Wallace, J. G., Kremling, K. A., Kovar, L. L. \& Buckler, E. S. Quantitative Genetics of the Maize Leaf Microbiome. Phytobiomes Journal 2, 208-224 (2018).

58. Wagner, M. R., Busby, P. E. \& Balint-Kurti, P. Analysis of leaf microbiome composition of near-isogenic maize lines differing in broad-spectrum disease resistance. New Phytol. 225, 2I52-2। 65 (2020).

59. Holm, J. B. et al. Ultrahigh-Throughput Multiplexing and Sequencing of $>500$-Base-Pair Amplicon Regions on the Illumina HiSeq 2500 Platform. mSystems 4, (2019).

60. de Muinck, E. J., Trosvik, P., Gilfillan, G. D., Hov, J. R. \& Sundaram, A. Y. M. A novel ultra high-throughput $16 \mathrm{~S}$ rRNA gene amplicon sequencing library preparation method for the Illumina HiSeq platform. Microbiome 5, 68 (2017).

6I. Weller, S. A., Elphinstone, J. G., Smith, N. C., Boonham, N. \& Stead, D. E. Detection of Ralstonia solanacearum strains with a quantitative, multiplex, real-time, fluorogenic PCR (TaqMan) assay. 
Appl. Environ. Microbiol. 66, 2853-2858 (2000).

62. Bowers, R. M. et al. Impact of library preparation protocols and template quantity on the metagenomic reconstruction of a mock microbial community. BMC Genomics 16, 856 (20I5).

63. Rinke, C. et al. Validation of picogram- and femtogram-input DNA libraries for microscale metagenomics. PeerJ 4, e2486 (2016).

64. Bustin, S. \& Huggett, J. qPCR primer design revisited. Biomol Detect Quantif I4, I 9-28 (20I7).

65. Pierce, K. E., Sanchez, J. A., Rice, J. E. \& Wangh, L. J. Linear-After-The-Exponential (LATE)-PCR: primer design criteria for high yields of specific single-stranded DNA and improved real-time detection. Proc. Natl. Acad. Sci. U. S. A. 102, 8609-86/4 (2005).

66. Sanchez, J. A., Pierce, K. E., Rice, J. E. \& Wangh, L. J. Linear-after-the-exponential (LATE)-PCR: an advanced method of asymmetric PCR and its uses in quantitative real-time analysis. Proc. Natl. Acad. Sci. U. S. A. I0I, 1933-1938 (2004).

67. Stergiopoulos, I. \& Gordon, T. R. Cryptic fungal infections: the hidden agenda of plant pathogens. Front. Plant Sci. 5, 506 (2014).

68. St Clair, D. A. Quantitative disease resistance and quantitative resistance Loci in breeding. Annu. Rev. Phytopathol. 48, 247-268 (2010).

69. Marotz, C. et al. Triplicate PCR reactions for I6S rRNA gene amplicon sequencing are unnecessary. Biotechniques 67, 29-32 (2019).

70. Rueden, C. T. et al. Image 2: Image for the next generation of scientific image data. BMC Bioinformatics I 8, 529 (2017).

7I. Rognes, T., Flouri, T., Nichols, B., Quince, C. \& Mahé, F. VSEARCH: a versatile open source tool for metagenomics. PeerJ 4, e2584 (2016).

72. Cole, J. R. et al. Ribosomal Database Project: data and tools for high throughput rRNA analysis. Nucleic Acids Res. 42, D633-42 (2014).

73. Nilsson, R. H. et al. The UNITE database for molecular identification of fungi: handling dark taxa and parallel taxonomic classifications. Nucleic Acids Res. 47, D259-D264 (2019).

74. R Core Team. R: A Language and Environment for Statistical Computing. (2019).

75. Wickham, H. ggplot2: Elegant Graphics for Data Analysis. (2016).

76. Wickham, H. Reshaping Data with the reshape Package. Journal of Statistical Software vol. 2 I I-20 (2007).

77. Daniels, M. J., Barber, C. E., Turner, P. C., Cleary, W. G. \& Sawczyc, M. K. Isolation of Mutants of Xanthomonas campestris pv. campestris Showing Altered Pathogenicity. Microbiology 130 , 2447-2455 (1984).

78. Dieterich, C. et al. The Pristionchus pacificus genome provides a unique perspective on nematode lifestyle and parasitism. Nat. Genet. 40, II93-I I 98 (2008).

79. Kurtz, Z., Mueller, C., Miraldi, E. \& Bonneau, R. SpiecEasi: Sparse Inverse Covariance for Ecological Statistical Inference. (2020).

80. Epskamp, S., Cramer, A. O. J., Waldorp, L. J., Schmittmann, V. D. \& Borsboom, D. qgraph: Network Visualizations of Relationships in Psychometric Data. Journal of Statistical Software vol. 48 I-I8 (20I2). 